\title{
VALUATION OF VIX DERIVATIVES
}

Javier Mencia and Entique sentana.

Documentos de Trabajo. N. 1232

\section{BANCODEESPANA}

\author{
Eurosistema
}


VALUATION OF VIX DERIVATIVES 
VALUATION OF VIX DERIVATIVES ${ }^{(*)}$

\author{
Javier Mencía
}

BANCO DE ESPAÑA

\title{
Enrique Sentana
}

CEMFI

$\left(^{*}\right)$ We are grateful to Dante Amengual, Torben Andersen, Max Bruche, Peter Carr, Rob Engle, Javier Gil-Bazo, loannis Paraskevopoulos, Francisco Peñaranda, Eduardo Schwartz, Neil Shephard and Dacheng Xiu, as well as seminar participants at Oxford MAN Institute, Universidad de Murcia, Universitat Pompeu Fabra, the SoFiE European Conference (Geneva, June 2009), the Finance Forum (Elche, November 2010) and the Symposium of the Spanish Economic Association (Madrid, December 2010) for helpful comments, discussions and suggestions. The input of an anonymous referee has also greatly improved the paper. Of course, the usual caveat applies. Financial support from the Spanish Ministry of Science and Innovation through grants ECO 2008-00280 and 2011-26342 (Sentana) is gratefully acknowledged. The views expressed in this paper are those of the authors, and do not reflect those of the Bank of Spain. Address for correspondence: Alcalá 48, 28014 Madrid, Spain, tel.: +34 91338 5414, fax: +34913386102. 
The Working Paper Series seeks to disseminate original research in economics and finance. All papers have been anonymously refereed. By publishing these papers, the Banco de España aims to contribute to economic analysis and, in particular, to knowledge of the Spanish economy and its international environment.

The opinions and analyses in the Working Paper Series are the responsibility of the authors and, therefore, do not necessarily coincide with those of the Banco de España or the Eurosystem.

The Banco de España disseminates its main reports and most of its publications via the INTERNET at the following website: http://www.bde.es.

Reproduction for educational and non-commercial purposes is permitted provided that the source is acknowledged.

C BANCO DE ESPAÑA, Madrid, 2012

ISSN: 1579-8666 (on line) 


\section{Abstract}

We conduct an extensive empirical analysis of VIX derivative valuation models before, during and after the 2008-2009 financial crisis. Since the restrictive mean reversion and heteroskedasticity features of existing models yield large distortions during the crisis, we propose generalisations with a time varying central tendency, jumps and stochastic volatility, and analyse their pricing performance, and implications for term structures of VIX futures and volatility «skews». We find that a process for the log of the observed VIX combining central tendency and stochastic volatility reliably prices VIX derivatives. We also uncover a significant risk premium that shifts the long-run volatility level.

Keywords: central tendency, stochastic volatility, jumps, term structure, volatility skews.

JEL classification: G13. 


\section{Resumen}

Realizamos un extenso análisis empírico de los modelos de valoración de los derivados sobre el VIX antes, durante y después de la crisis financiera de 2008-2009. Como las características restrictivas de reversión a la media y heteroscedasticidad de los modelos existentes generan grandes distorsiones durante la crisis, proponemos generalizaciones con una tendencia central cambiante en el tiempo, saltos y volatilidad estocástica, analizando la adecuación de sus precios y sus implicaciones sobre la estructura temporal de los futuros del VIX y las curvas de volatilidad implícita. Nuestros resultados indican que un proceso para el logaritmo del VIX que combina una tendencia central y volatilidad estocástica valora adecuadamente los derivados sobre el VIX. Asimismo, hallamos una prima de riesgo significativa que desplaza el nivel de la volatilidad a largo plazo.

Palabras clave: tendencia central, volatilidad estocástica, saltos, estructura temporal, curvas de volatilidad implícita

Códigos JEL: G13. 


\section{Introduction}

It is now widely accepted that the volatility of financial assets changes stochastically over time, with fairly calmed phases being followed by more turbulent periods of uncertain length. For financial market participants, it is of the utmost importance to understand the nature of those variations because volatility is a crucial determinant of their investment decisions. Although many model-based and model-free volatility measures have been proposed in the academic literature (see Andersen, Bollerslev, and Diebold, 2009, for a recent survey), the Chicago Board Options Exchange (CBOE) volatility index, widely known by its ticker symbol VIX, has effectively become the standard measure of volatility risk for investors in the US stock market. The goal of the VIX index is to capture the volatility (i.e. standard deviation) of the S\&P500 over the next month implicit in stock index option prices. Formally, it is the square root of the risk neutral expectation of the integrated variance of the S\&P500 over the next 30 calendar days, reported on an annualised basis. Despite this rather technical definition, both financial market participants and the media pay a lot of attention to its movements. To some extent, its popularity is due to the fact that VIX changes are negatively correlated to changes in stock prices. The most popular explanation is that investors trade options on the S\&P500 to buy protection in periods of market turmoil, which increases the value of the VIX. In fact, as Andersen and Bondarenko (2007) and many others show, the VIX almost uniformly exceeds realised volatility because investors are on average willing to pay a sizeable premium to acquire a positive exposure to future equity-index volatility. For that reason, some commentators refer to it as the market's fear gauge, even though a high value does not necessarily imply negative future returns.

But apart from its role as a risk indicator, nowadays it is possible to directly invest in volatility as an asset class by means of VIX derivatives. Specifically, on March 26, 2004, trading in futures on the VIX began on the CBOE Futures Exchange (CFE). They are standard futures contracts on forward 30-day implied vols that cash settle to a special opening quotation (VRO) of the VIX on the Wednesday that is 30 days prior to the 3rd Friday of the calendar month immediately following the expiring month. Further, on February 24, 2006, European-style options on the VIX index were also launched on the CBOE. Like VIX futures, they are cash settled according to the difference between 
the value of the VIX at expiration and their strike price. Importantly, they can also be interpreted as options on VIX futures, which one can exploit to simplify their valuation and avoid compounding errors made in valuing futures. VIX options and futures are among the most actively traded contracts at CBOE and CFE, averaging close to 260,000 contracts combined per day in 2010, with much larger volumes on certain days. ${ }^{1}$ One of the main reasons for the high interest in these products is that VIX derivative positions can be used to hedge the risks of investments in the S\&P500 index. Szado (2009) finds that such strategies do indeed provide significant protection, especially in downturns. Moreover, by holding VIX derivatives investors can achieve exposure to S\&P500 volatility without having to delta hedge their S\&P500 option positions with the stock index. As a result, it is often cheaper to be long in out-of-the-money VIX call options than to buy out-of-the-money puts on the S\&P500. Due to this possibility, VIX options are the only asset in which open interest is highest for out-of-the-money call strikes (Rhoads, 2011).

Although these new assets certainly offer additional investment and hedging opportunities for financial market participants, their correct use requires reliable valuation models that adequately capture the features of the underlying volatility index. In turn, the empirical performance of those valuation models can shed some light on the stochastic process for the VIX, and stock market volatility more generally, which is of interest to academic researchers. Moreover, the prices of volatility derivatives contain valuable information about the views financial market participants hold about the future, and we need reliable valuation models to make the correct inferences in a world with risk averse agents.

Somewhat surprisingly, several theoretical approaches to price VIX derivatives appeared in the academic literature long before they could be traded. Specifically, Whaley (1993) priced volatility futures assuming that the observed volatility index on which they are written follows a Geometric Brownian Motion (GBM). As a result, his model does not allow for mean reversion in the VIX, which, as we shall see, seems to be at odds with the recent empirical evidence. ${ }^{2}$ The two most prominent mean reverting models

\footnotetext{
${ }^{1}$ For comparison purposes, this value represents about $37 \%$ of the average daily volume of S\&P500 options, which was 695,000 in 2010. However, the volume of VIX derivatives is growing at a much faster rate. Specifically, the volume of VIX options already represented $52 \%$ of the volume of S\&P500 options during the first ten months of 2011. In this sense, Rhoads (2011) points out that the volume traded is executed over a smaller time frame (7:20 am - 3:15 pm Central Time) than for other derivatives markets.

${ }^{2}$ The stationarity of volatility seems to depend on the historical period considered. Schwert (1990) and Pagan and Schwert (1990) find strong evidence for a unit root in stock volatility if the data spans the 1930's. In contrast, Schwert (2011) convincingly argues that during the 2008-2009 crisis volatility exhibits more mean reversion than in the past.
} 
proposed so far for volatility indices have been the square root process (SQR) considered by Grünbichler and Longstaff (1996), and the log-normal Ornstein-Uhlenbeck (LOU) process analysed by Detemple and Osakwe (2000).

Several authors have previously looked at the empirical performance of these pricing models for VIX derivatives. In particular, Zhang and Zhu (2006) study the empirical validity of the SQR model by first estimating its parameters from VIX historical data, and then assessing the pricing errors of VIX futures implied by those estimates. Following a similar estimation strategy, Dotsis, Psychoyios, and Skiadopoulos (2007) also use VIX futures data to evaluate the gains of adding jumps to a SQR diffusion. In addition, they estimate a GBM process. Surprisingly, this model yields reasonably good results, but the time span of their sample is perhaps too short for the mean reverting features of the VIX to play any crucial role. In turn, Wang and Daigler (2011) compare the empirical fit of the SQR and GBM models using data on options written on the VIX. They also find evidence supporting the GBM assumption. However, one could alternatively interpret their findings as evidence in favour of the LOU process, which also yields the Black (1976) option formula if the underlying instrument is a VIX futures contract, but at the same time is consistent with mean reversion.

Despite this empirical evidence, both the SQR and the LOU processes show some glaring deficiencies in capturing the strong persistence of the VIX, which produces large and lasting deviations of this index from its long run mean. In contrast, the implicit assumption in those models is that this volatility index mean reverts at a simple, nonnegative exponential rate. Such a limitation becomes particularly apparent during bearish stock markets, in which volatility measures such as the VIX typically experience large increases and remain at high levels for long periods. Arguably, the apparent success of those models is to a large extent due to the fact that the sample periods considered in the existing studies only cover the relatively long and quiet bull market that ended in the summer of 2007.

In this context, the initial objective of our paper is to study the empirical ability of existing mean-reverting models for volatility indices to price derivatives on the VIX over a longer sample span that includes data before, during and after the unprecedented recent financial crisis, which provides a unique testing ground for our study. To do so, 
we use an extensive database that comprises all futures and European option prices on the VIX from February 2006, when options were introduced, until December 2010. As a result, we can study whether the SQR and LOU models are able to yield reliable inand out-of-sample prices in a variety of market circumstances.

Our findings indicate that although a LOU process for the VIX provides a better fit than a SQR model, especially for VIX options, its performance deteriorates during the market turmoil of the second part of our sample. For that reason, we consider an extension of the SQR model advocated by Bates (2012) among others, which allows for a time varying central tendency in the mean as well as stochastic volatility that is unspanned by the VIX. Similarly, we generalise the LOU process by proposing several novel but empirically relevant extensions: a time-varying central tendency in the mean, jumps, and stochastic volatility. A central tendency, which was first introduced by Jegadeesh and Pennacchi (1996) and Balduzzi, Das, and Foresi (1998) in the context of term structure models, allows the "average" volatility level to be time-varying, while stochastic volatility permits a changing dispersion for the (log) volatility index, and together with jumps, introduces non-normality in its conditional distribution. Importantly, we study the role that risk premia plays in reconciling the dynamics of the VIX with the prices of VIX derivatives in order to determine the existence of economically important systematic risks.

We estimate the SQR and LOU models by maximum likelihood. However, a closedform expression for the likelihood is not available for many of the extensions. As a consequence, following Trolle and Schwartz (2009) among others, we specify our extensions in state space form and calibrate their parameters by pseudo maximum likelihood. We use both derivative prices and historical observations on the VIX itself since we want to estimate both real and risk-neutral measures. In order to compute the theoretical derivatives prices, we often need to invert the conditional characteristic function using Fourier methods (see Carr and Madan, 1999, and Amengual and Xiu, 2012).

To validate the models, we analyse the discrepancies between actual and theoretical derivatives prices. But we also go beyond pricing errors, and analyse the implications of the aforementioned extensions for the term structures of VIX futures, and the option volatility "skews", all of which are of considerable independent interest. Since we combine futures and options data, we can also assess which of those additional features is more relevant for pricing futures, and which one is more important for options. 
Importantly, our approach differs from Sepp (2008), who prices VIX derivatives by using data on the S\&P500 only. Although the relevant distribution of future values of the VIX conditional on current information might arguably depend not only on the current level of the VIX but also on the value of the S\&P500, it seems odd to completely neglect the information content of contemporaneously observed VIX values. This is particularly relevant in view of the fact that one cannot reproduce the spot VIX index by using the model proposed by Sepp (2008) for the purposes of obtaining the required S\&P500 option values that feed in the closed-form CBOE formula for this volatility index. ${ }^{3}$ In order to avoid compounding valuation errors, therefore, we prefer to treat the current VIX level as our "sufficient statistic", and to treat VIX futures likewise for the purposes of valuing options, instead of assigning that role to the S\&P500. The same assumption has recently been made by Song and Xiu (2012). Ideally, though, one would like to use both, but we leave this for future research. In this sense, it is important to emphasise that the univariate stochastic processes that we consider for the VIX are not necessarily incompatible with models for S\&P500 options capable of reproducing this observed volatility index.

Our analysis can also shed some light on the long-lasting debate surrounding the modelling and stationarity of the (instantaneous) volatility of this broad stock market index. In particular, given the relationship between the observed VIX and the unobserved integrated volatility of the S\&P500, our results imply that the sophisticated stochastic volatility models that many researchers and practitioners use for valuing S\&P options should probably allow for a slower mean reversion than usually considered, as well as for time-varying volatility of volatility.

The rest of the paper is organised as follows. We describe the data in Section 2, and explain our estimation strategy in Section 3. Then, we assess the empirical performance of existing models in Section 4 and consider our proposed extensions in Section 5. Finally, we conclude in Section 6. Auxiliary results are gathered in several Appendices.

\footnotetext{
${ }^{3} \mathrm{~A}$ relevant analogy would be the use of a model for the dividends and risk premia of the 500 individual stocks that constitute the index for the purposes of valuing S\&P500 derivatives discarding the information in the index itself, even though any such multivariate model would very likely fail to reproduce the value of the index.
} 


\section{Preliminary data analysis}

\subsection{The CBOE Volatility Index}

VIX was originally introduced in 1993 to track the Black-Scholes implied volatilities of options on the S\&P100 with near-the-money strikes (see Whaley, 1993). The CBOE redefined the index in 2003, renamed the original index as VXO, and released a time series of daily closing prices starting in January 1990 (see Carr and Wu, 2006). Nowadays, VIX is computed in real time using as inputs the mid bid-ask market prices for most calls and puts on the S\&P500 index for the front month and the second month expirations with at least 8 days left (see CBOE, 2009). ${ }^{4}$ Since VIX is expressed in annualised terms, investors typically divide it by $16(=\sqrt{256})$ to gauge the expected size of the daily movements in the stock market implied by this index (see Rhoads, 2011).

Figure 1a displays the entire historical evolution of the VIX. Between January 1990 and December 2010 its average closing value was 20.4. As other volatility measures, though, it is characterised by swings from low to high levels, with a temporal pattern that shows mean reversion over the long run but displays strongly persistent deviations from the mean during extended periods. The lowest closing price (9.31) corresponds to December 22, 1993. Figure 1b, which focuses on the sample period in our derivatives database, shows that volatility was also remarkably low between February 2006 and July 2007, with values well below 20. During this period, the lowest value was 9.89 on January 24, 2007, in what some have called "the calm before the storm". Indeed, although the Dow Jones Industrial Average closed above 14,000 for the first time in history in the summer of 2007, some warning signals were observed around this period. In particular, on June 22, 2007, Bear Stearns pledged up to $\$ 3.2$ billion in loans to bail out one of its hedge funds, which was collapsing due to bad bets on subprime mortgages. Moreover, on July 18, 2007, this investment bank disclosed that its two subprime hedge funds had lost all of their value, and one day later Fed Chairman Ben Bernanke warned the US Senate Banking Committee that subprime losses could top $\$ 100$ billion. Perhaps not surprisingly, over the following year VIX increased to values between 20 and 35. Finally, in the autumn of 2008 it reached unprecedented levels. In particular, the largest

\footnotetext{
${ }^{4}$ Currently, CBOE applies the VIX methodology to 3-month options on the S\&P500 (VXV), as well as 1-month options on the most important US stock market indices: DJIA (VXD), S\&P100 (VXO), Nasdaq-100 (VXN) and Russell 2000 (RVX). They also construct analogous short term volatility indices for Crude Oil (OVX), Gold (GVZ), Soybean (SIV), Corn (CIV) and the US \$/€exchange rate (EVZ).
} 
historical closing price (80.86) took place on November 20, 2008, although on October 24 the VIX reached an intraday value of 89.53. After this peak, VIX followed a decreasing trend over the following months until the beginning of April, 2010, when the Greek debt crisis started worsening. These markedly different regimes offer a very interesting testing ground to analyse the performance of valuation models for volatility derivatives. In particular, we can assess if the models calibrated with pre-crisis data perform well under the extreme conditions of the 2008-2009 financial crisis.

In order to characterise the time series dynamics of the VIX, we have estimated several ARMA models using the whole VIX historical observations from 1990 to 2010 (see French, Schwert, and Stambaugh, 1987, for a related analysis). Figure 2a compares the sample autocorrelations of the log VIX with those implied by the estimated models. There is clear evidence of high persistence, with a first-order autocorrelation above 0.98 and a slow rate of decay for higher orders. Consequently, an AR(1) model seems unable to capture the shape of the sample correlogram. An alternative illustration of the failure of this model is provided by the presence of positive partial autocorrelations of orders higher than one, as Figure $2 \mathrm{~b}$ confirms. Therefore, it is necessary to introduce a moving average component to take into account this feature. An $\operatorname{ARMA}(1,1)$ model, though, only offers a slight improvement. In contrast, an $\operatorname{ARMA}(2,1)$ model turns out to yield autocorrelations and partial autocorrelations that are much closer to the sample values. As we shall see below, our preferred continuous time models have $\operatorname{ARMA}(2,1)$ representations in discrete time. Importantly, this result is not due to the behaviour of the VIX during the financial crisis, since an $\operatorname{ARMA}(2,1)$ is also necessary to capture the autocorrelation profile when we only consider data from 1990 until the summer of 2007.

We have also analysed the presence of time varying features in the volatility of the log VIX. In particular, we have tested for GARCH effects in the residuals of the $\operatorname{ARMA}(2,1)$ estimation. ${ }^{5}$ Interestingly, we can easily reject the conditional homoskedasticity of the $\log$ VIX at all conventional levels. As we shall in Section 5.4, the volatility of our preferred continuous time specification closely matches the volatility of an $\operatorname{ARMA}(2,1)$ $\operatorname{GARCH}(1,1)$ model.

\footnotetext{
${ }^{5}$ Following Demos and Sentana (1998), we carry out a one-sided LM test of conditional homoskedasticity against GARCH as $T \tilde{R}^{2}$ from the regression of the squared $\operatorname{ARMA}(2,1)$ residuals on a constant and the Riskmetrics volatility estimate (see RiskMetrics Group, 1996).
} 


\subsection{VIX derivatives}

Our sample contains daily closing bid-ask mid prices of futures and European put and call options on the VIX, which we downloaded from Bloomberg. We apply several filters to ensure the reliability of the data that we finally use. Following Dumas, Fleming, and Whaley (1998), we exclude derivatives with fewer than six days to expiration due to their illiquidity. In addition, we only retain those prices for which open interests and volumes are available. We consider the entire history of these series since options were introduced in February 2006 until December 2010. In terms of maturity, we have data on all the contracts with expirations between March 2006 and May (July) 2011 for options (futures). CFE may list futures for up to 9 near-term serial months, as well as 5 months on the February quarterly cycle associated to the March quarterly cycle for options on the S\&P500. In turn, CBOE initially lists some in-, at- and out-of-the-money strike prices, and then adds new strikes as the VIX index moves up or down. Generally, the options expiration dates are up to 3 near-term months plus up to 3 additional months on the February quarterly cycle. ${ }^{6}$

All in all, we have 8,665 and 87,870 prices of futures and options, respectively. Of those option prices, 58,099 correspond to calls and 29,771 to puts. We have between 4 and 6 daily futures prices during 2006; afterwards, 8 prices per day become available on average. The number of option prices per day is also smaller at the beginning of the sample, but it tends to stabilise in 2007 at around 20 for puts and 40 for calls. The number of call prices per day increases again after mid 2009 and is greater than 60 at the end of the sample. We proxy for the riskless interest rate by using the daily Eurodollar rates at 1-week, 1, 3 and 6 months and 1 year, which we interpolate to match the maturities of the futures and option contracts that we observe. ${ }^{7}$

Figure 3a shows the evolution of the VIX term structure implicit in VIX futures. Futures prices remained low albeit above the volatility index until mid 2007, which suggests that market participants perceived that the VIX was too low during those

\footnotetext{
${ }^{6}$ VIX futures contracts have a multiplier of 1000, while VIX option contracts have a multiplier of 100. This means that the value of the VIX futures contract is determined by multiplying $\$ 1,000$ times the quoted futures price. On March 2, 2009, the CBOE introduced mini-VIX futures, which have a multiplier of 100 (see Rhoads, 2011).

${ }^{7}$ Given that Eurodollar and Libor rates are very similar, the results are unlikely to be sensitive to this choice. As usual in the option pricing literature, we assume constant interest rates in our models. Although interest rates are of course not constant in practice, we have checked that their changes are not significantly correlated with changes in the VIX.
} 
years. For instance, on October 12, 2006, the VIX was 11.09, while the price of VIX futures expiring on February 14, 2007, was 15.72. The spot price reflected the expected volatility for the period of October 12 to November 11, while the futures price reflected the expected volatility for the period of February 14 to March 16, 2007. Figure 3a clearly confirms that there was an initial increase in the summer of 2007 and a substantial level shift in the last quarter of 2008. The negative slope during the latter period, though, indicates that the market did not expect the VIX to remain at such high values forever, a fact already highlighted by Schwert (2011). Eventually, this prediction turned out to be on the winning side and futures prices significantly came down until April 2010, right before the beginning of the European sovereign debt crisis.

Figures $3 \mathrm{~b}$ and $3 \mathrm{c}$ show the average implied volatility skews implicit in VIX option prices for different times to maturity. We consider low volatility days on Figure $3 \mathrm{~b}$ and high volatility days on Figure 3c. We define low (high) volatility days as those in which the implied volatility of one-month at-the-money options remain below (above) their average value over the sample. Interestingly, we observe a consistent pattern of implied volatility smirks with positive slope regardless of the time to maturity and volatility of the VIX. There only seems to be a small change in slope for deep in-the-money calls (or out-of-the-money puts). Therefore, any pricing model must adequately capture these empirical features. Finally, although Figures $3 \mathrm{~b}$ and $3 \mathrm{c}$ show that option prices were higher for longer maturities on average, this is not necessarily the case on all the days in the sample. In particular, option prices decreased with maturity at the peak of the financial crisis (November 2008) because the market expected a fall in the volatility of the VIX.

\section{Pricing and estimation strategy}

We assume that there is a risk free asset with instantaneous rate $r$. Let $V(t)$ be the VIX value at time $t$. We define $F(t, T)$ as the actual price of a futures contract on $V(t)$ that matures at $T>t$. Similarly, we will denote the prices at $t$ of call and put options maturing at $T$ with strike price $K$ by $c(t, T, K)$ and $p(t, T, K)$, respectively. Importantly, since the VIX index is a risk neutral volatility forecast, not a directly traded asset, there is no cost of carry relationship between the price of the futures and the VIX (see Grünbichler and Longstaff, 1996, for more details). There is no convenience yield either, as in the 
case of futures on commodities. Therefore, absent any other market information, VIX derivatives must be priced according to some model for the risk neutral evolution of the VIX. This situation is similar, but not identical, to term structure models.

Let $\mathcal{M}$ index the asset pricing models that we consider. Then, the theoretical futures price implied by model $\mathcal{M}$ will be:

$$
F_{\mathcal{M}}(t, T, V(t), \phi)=E_{\mathcal{M}}^{Q}[V(T) \mid I(t), \phi],
$$

where $Q$ indicates that the expectation is evaluated at the risk neutral measure, $\phi$ is the vector of free parameters of model $\mathcal{M}$, and $I(t)$ denotes the information available at time $t$, which includes $V(t)$ and its past values.

We can analogously express the theoretical value of a European call option with strike $K$ and maturity at $T$ under this model as

$$
c_{\mathcal{M}}[t, T, K, F(t, T), \phi]=\exp (-r \tau) E_{\mathcal{M}}^{Q}[\max (V(T)-K, 0) \mid I(t), \phi],
$$

where $\tau=T-t$. Nevertheless, we can exploit the fact that $V(T)=F(T, T)$ to price calls using the futures contract that expires on the same date as the underlying instrument, instead of the actual volatility index. In this way, we make sure that the pricing errors of options are not caused by distortions in our futures valuation formulas. Similarly, European put prices $p(t, T, K)$ under $\mathcal{M}$ can be easily obtained from the put-call-forward parity relationship

$$
p_{\mathcal{M}}[t, T, K, F(t, T), \phi]=c_{\mathcal{M}}[t, T, K, F(t, T), \phi]-\exp (-r \tau)[F(t, T)-K]
$$

We use futures and options prices, as well as data on the VIX index, which allows us to estimate the risk premia implicit in the market of VIX derivatives. We estimate the parameters by maximum likelihood for the models whose likelihood is known in closed form, and by pseudo maximum likelihood for the remaining ones. In both cases, we assume the existence of pricing errors such that

$$
\begin{gathered}
F(t, T)=F_{\mathcal{M}}(t, T, V(t), \phi)+\xi_{f t}+\varepsilon_{f t, T}, \\
c(t, T, K)=c_{\mathcal{M}}[t, T, K, F(t, T), \phi]+\varpi(M(t, T))\left[\left(1-\eta_{o}\right) \xi_{o t}+\eta_{o} \varepsilon_{c t, T}(K)\right], \\
p(t, T, K)=p_{\mathcal{M}}[t, T, K, F(t, T), \phi]+\varpi(M(t, T))\left[\left(1-\eta_{o}\right) \xi_{o t}+\eta_{o} \varepsilon_{p t, T}(K)\right],
\end{gathered}
$$

where $\varepsilon_{f t, T} \sim N\left(0, \sigma_{f \varepsilon}^{2}\right), \varepsilon_{c t, T}(K) \sim N(0,1)$ and $\varepsilon_{p t, T}(K) \sim N(0,1)$ are independent and iid over time and across strikes and maturities, while $\xi_{f t} \sim N\left(0, \sigma_{f \xi}^{2}\right)$ and $\xi_{\text {ot }} \sim N(0,1)$ 
are also orthogonal and iid over time but common for all futures and options, respectively. Thus, we decompose pricing errors into common and idiosyncratic terms. Thanks to this parametrisation, we allow for contemporaneous correlation between the pricing errors. As noted by Bates (2000), ignoring this feature would affect the relative weighting that the options and futures would receive in the estimation. In contrast, the inclusion of common factors avoids overestimating the amount of truly independent information in the data. ${ }^{8}$ In addition, we parametrise the variances of option pricing errors by means of the quadratic function

$$
\varpi^{2}(M(t, T))=\sigma_{a}+\sigma_{b}\left(M(t, T)-\sigma_{c}\right)^{2}
$$

where $M(t, T)=\log (K / F(t, T))$ measures the moneyness of the option. In this way, we avoid giving undue weight to higher priced or in-the-money options whose pricing errors are higher simply because of their larger scale. Importantly, we also employ different parameters for the pricing error variances of futures and options, thereby implicitly adjusting their relative weight in the likelihood.

We can write the log likelihood at a particular time $t$ as

$$
l\left[\mathbf{o}_{t}, \mathbf{f}_{t}, V(t) \mid I(t-1)\right]=l_{o}\left(\mathbf{o}_{t} \mid \mathbf{f}_{t}, V(t), I(t-1)\right)+l_{f}\left(\mathbf{f}_{t} \mid V(t), I(t-1)\right)+l_{v}[V(t) \mid I(t-1)],
$$

where $\mathbf{o}_{t}$ and $\mathbf{f}_{t}$ are, respectively, the set of option and futures prices traded at $t$, $l_{o}\left(\mathbf{o}_{t} \mid \mathbf{f}_{t}, V(t), I(t-1)\right)$ is the log density of the option prices conditional on futures prices and VIX, $l_{f}\left(\mathbf{f}_{t} \mid V(t), I(t-1)\right)$ is the log likelihood of the futures prices conditional on the volatility index, and $l_{v}[V(t) \mid I(t-1)]$ is the log density of the volatility index. ${ }^{9}$ Thus, the log likelihood reflects that we price futures conditional on the value of the VIX, in the same we as we price options conditional on the value of the futures. In addition, we take into account the contribution of the VIX to the log likelihood in order to obtain the parameters under the real measure.

A non-trivial advantage of our estimation method over traditional calibration procedures is that we do not have to treat the model parameters as deterministic functions of time. In addition, we can also obtain standard errors for our parameter estimators, which can in turn be used to conduct formal hypothesis tests. Furthermore, we can

\footnotetext{
${ }^{8}$ Nevertheless, empirical results without the common factors $\xi_{f t}$ and $\xi_{o t}$, which are available from the authors upon request, yield qualitatively similar results.

${ }^{9}$ The usefulness of this additive decomposition is limited, though, because most model parameters affect more than one component.
} 
exploit the relationship between actual and risk-neutral measures to estimate prices of risk (see Appendices B and C for details).

We consider two estimation samples that correspond to two distinct volatility phases described in Section 2: until 15-Aug-2008 and full sample. We use the first sample to evaluate the out-of-sample empirical fit of the models. ${ }^{10}$ Thus, we can assess model performance following a major volatility increase in global stock markets.

\section{Existing one-factor models}

\subsection{Model specification}

We first compare the two mean-reverting volatility models that have been used so far in the literature: the square root and the log Ornstein-Uhlenbeck processes. As we mentioned before, Grünbichler and Longstaff (1996) proposed the square root process (SQR) to model a standard deviation index. This model, which was used by Cox, Ingersoll, and Ross (1985) for interest rates and Heston (1993) for the instantaneous variance of stock prices, satisfies the diffusion

$$
d V(t)=\kappa^{P}\left[\bar{\theta}^{P}-V(t)\right] d t+\sigma \sqrt{V(t)} d W^{P}(t),
$$

where $W^{P}(t)$ is a Brownian motion under the real measure. Following Dai and Singleton (2000), we specify a price of risk that is proportional to the instantaneous volatility. Specifically, we assume that $\Lambda_{v}(t)=\varsigma \sqrt{V(t)}$, so that $d W^{Q}(t)=d W^{P}(t)+\varsigma \sqrt{V(t)} d t$. Then, it is straightforward to show that $V(t)$ satisfies the square root diffusion

$$
d V(t)=\kappa[\bar{\theta}-V(t)] d t+\sigma \sqrt{V(t)} d W^{Q}(t)
$$

under the equivalent risk neutral measure, where $\kappa=\kappa^{P}+\sigma \varsigma$ and $\bar{\theta}=\kappa^{P} \bar{\theta}^{P} / \kappa$.

As is well known, the risk-neutral distribution of $2 c V(T)$ given $V(t)$ is a non-central chi-square with $\nu=4 \kappa \bar{\theta} / \sigma^{2}$ degrees of freedom and non-centrality parameter $\psi=$ $2 c V(t) \exp (-\kappa \tau)$, where

$$
c=\frac{2 \kappa}{\sigma^{2}(1-\exp (-\kappa \tau))} .
$$

\footnotetext{
${ }^{10}$ We analyse out-of-sample performance using the parameter estimates obtained in-sample. We also use the current value of the VIX to compute futures prices and the current value of futures prices to compute option prices, just as a financial market participant would do in real time. In addition, in the models with more than one factor we follow the standard procedure of filtering the additional factor using the past evolution of the VIX and its derivatives with the in-sample estimates.
} 
As a result, the price of the futures contract (1) can be expressed in this case as

$$
F_{S Q R}(t, T, V(t), \kappa, \bar{\theta}, \sigma)=\bar{\theta}+\exp (-\kappa \tau)[V(t)-\bar{\theta}] .
$$

We can interpret $\bar{\theta}$ as the long-run mean of $V(t)$, since the conditional expected value of the volatility index converges to $\bar{\theta}$ as $\tau$ goes to infinity. In addition, $\kappa$ is usually interpreted as a mean-reversion parameter because the higher it is, the more quickly the process reverts to its long run mean. ${ }^{11}$ Interestingly, the SQR model introduces stochastic volatility because the conditional variance of the VIX implied by this model is an affine function of $V(t)$ (see Appendix D).

The call price formula (2) for this model becomes

$$
\begin{aligned}
c_{S Q R}(t, T, K, \kappa, \bar{\theta}, \sigma)= & V(t) \exp (-(\kappa+r) \tau)\left[1-F_{N C 2}(2 c K ; \nu+4, \psi)\right] \\
& +\bar{\theta}[1-\exp (-\kappa \tau)] \exp (-r \tau)\left[1-F_{N C 2}(2 c K ; \nu+2, \psi)\right] \\
& -K \exp (-r \tau)\left[1-F_{N C 2}(2 c K ; \nu, \psi)\right],
\end{aligned}
$$

where $F_{N C 2}(\cdot ; \nu, \psi)$ is the cumulative distribution function (cdf) of a non-central chisquare distribution with $\nu$ degrees of freedom and non-centrality parameter $\psi$. Hence, it is straightforward to express the call price as a function of $F(t, T)$ by exploiting the relationship between futures prices and $V(t)$ in (4).

Subsequently, Detemple and Osakwe (2000) considered the log-normal OrnsteinUhlenbeck (LOU) diffusion:

$$
d \log V(t)=\kappa\left[\bar{\theta}^{P}-\log V(t)\right] d t+\sigma d W^{P}(t) .
$$

If once again we follow Dai and Singleton (2000) in specifying the price of risk as $\Lambda_{v}(t)=$ $\varsigma$, then $V(t)$ will satisfy the LOU diffusion

$$
d \log V(t)=\kappa[\bar{\theta}-\log V(t)] d t+\sigma d W^{Q}(t)
$$

under the risk neutral measure, where $\bar{\theta}=\bar{\theta}^{P}-\sigma \varsigma / \kappa$

As is well known, this model implies that $\log V(t)$ would follow a conditionally homoskedastic Gaussian AR(1) process if sampled at equally spaced discrete intervals. More generally, the conditional risk-neutral distribution of $\log V(T)$ given $V(t)$ would be Gaussian with mean

$$
\mu(t, \tau)=\bar{\theta}+\exp (-\kappa \tau)[\log V(t)-\bar{\theta}]
$$

\footnotetext{
${ }^{11}$ As remarked by Hansen and Scheinkman (2009), the density of this distribution will be positive at $V(T)=0$ if the Feller condition $2 \kappa \bar{\theta} \geq \sigma^{2}$ is violated.
} 
and variance

$$
\varphi^{2}(\tau)=\frac{\sigma^{2}}{2 \kappa}[1-\exp (-2 \kappa \tau)]
$$

As in the SQR process, $\bar{\theta}$ and $\kappa$ can be interpreted as the long-run mean and meanreversion parameters, respectively, but now it is the log of $V(t)$ that mean reverts to $\bar{\theta}$. And although (6) shows that the LOU model is homoskedastic for the log VIX, it can be shown that the process followed by the VIX is heteroskedastic due to Jensen's inequality (see Appendix D for more details). In this context, it is straightforward to show that the futures price is

$$
F_{L O U}(t, T, V(t), \kappa, \bar{\theta}, \sigma)=\exp \left(\mu(t, \tau)+0.5 \varphi^{2}(\tau)\right)
$$

while the call price can be expressed as

$$
\begin{aligned}
c_{L O U}(t, T, K, \kappa, \bar{\theta}, \sigma)= & \exp (-r \tau) F(t, T) \Phi\left[\frac{\log (F(t, T) / K)+\frac{1}{2} \varphi^{2}(\tau)}{\varphi(\tau)}\right] \\
& -K \exp (-r \tau) \Phi\left[\frac{\log (F(t, T) / K)-\frac{1}{2} \varphi^{2}(\tau)}{\varphi(\tau)}\right]
\end{aligned}
$$

if we take the futures contract as the underlying instrument, where $\Phi(\cdot)$ denotes the standard normal cdf. This is the well known Black (1976) formula, although in this case the implied volatility $\varphi(\tau)$ in (6) is not constant across maturities. In this sense, the pricing formula proposed by Whaley (1993) based on a geometric Brownian motion can also be expressed as (7) if $\varphi(\tau)$ is taken as a constant irrespective of $\tau$.

\subsection{Empirical performance}

We have estimated the parameters of these two models over the two sample periods described at the end of Section 3. Table 1 reports the in- and out-of-sample root mean square pricing errors (RMSE). We observe that the SQR model yields larger in-sample price distortions than the LOU model. Those distortions remain in the out-of-sample period. We have re-estimated the two models with VIX and futures data only to check that the use of option data in the estimation of the parameters is not driving the results. We have found that the LOU model still yields a better performance in that case.

Table 2 reports the parameter estimates that we obtain under the real measure, as well as the parameters of the pricing error variances. Although the values are not directly comparable because volatility is expressed in levels in the SQR model and in logs in the LOU model, in both cases the mean reversion parameter $\kappa$ is quite sensitive to the sample 
period used for estimation purposes (see footnote 2 and references therein). In contrast, the volatility parameter $\sigma$ is more stable for the LOU process, probably because the log transformation is more appropriate to capture the distortions produced by the large movements of the VIX that took place at the end of our sample. Table 2 also shows that the parameters of the quadratic function (3) are highly significant. Therefore, allowing for different variances of pricing errors across strikes seems to be relevant in ensuring that higher priced options do not receive undue weight.

An alternative, more illustrative way to assess the validity of the LOU model is by considering the implied volatilities obtained with the Black (1976) formula (7). As we mentioned before, the implied volatility of the LOU process is constant for different degrees of moneyness, but not across different maturities because (6) depends on $\tau$. Hence, if this model were correct then we should obtain constant implied volatilities for a given maturity regardless of moneyness. As we have seen in Figures $3 \mathrm{~b}$ and $3 \mathrm{c}$, this is not at all the case. In fact, we generally observe that implied vols have a positive moneyness slope. In addition, the comparison of these figures suggest that the volatility of the log VIX is not constant over time, as the LOU model assumes. In this sense, the LOU process is not only unable to generate the observed volatility skews, but it also fails to capture the average level of implied volatilities. As we will see in Section 5.5, though, the SQR model generates volatility skews, but they have the opposite slope to the actual skews in Figures 3b and 3c.

\section{Extensions}

\subsection{Model specification}

The results of the previous section indicate that a LOU process offers a better empirical fit than a SQR process. Unfortunately, their performance tends to deteriorate during the recent financial crisis. For that reason, in this section we explore several extensions to those models. For the sake of brevity, though, we focus on the risk-neutral measure and the prices of risk. Further details can be found in Appendices B and C.

Specifically, we extend the SQR model by considering the concatenated SQR (CSQR) proposed by Bates (2012) among others:

$$
d V(t)=\kappa[\theta(t)-V(t)] d t+\sigma \sqrt{V(t)} d W_{v}^{Q}(t)
$$


with

$$
d \theta(t)=\bar{\kappa}[\bar{\theta}-\theta(t)] d t+\bar{\sigma} \sqrt{\theta(t)} d W_{\theta}^{Q}(t) .
$$

We specify the prices of risk that link the Wiener processes under P and Q as

$$
\begin{aligned}
& d W_{v}^{Q}(t)=d W_{v}^{P}(t)+\varsigma \sqrt{V(t)} d t \\
& d W_{\theta}^{Q}(t)=d W_{\theta}^{P}(t)+\bar{\varsigma} \sqrt{\theta(t)} d t,
\end{aligned}
$$

where $W_{v}^{Q}(t)$ and $W_{\theta}^{Q}(t)$ are independent Brownian motions. These specifications ensure that the VIX follows the same process under the real measure. As we mentioned before, an important deficiency of previously existing models is that they assume that the volatility index mean reverts at a simple, non-negative exponential rate, which is in fact 0 in the GBM model proposed by Whaley (1993). However, the long, persistent swings in the VIX in Figure 1a suggest that we need to allow for more complex dynamics. In this sense, the CSQR allows the VIX to revert towards a central tendency, which in turn fluctuates stochastically over time around a long run mean $\bar{\theta}$. As a consequence, the conditional mean of $V(T)$ is a function of the distance between $V(t)$ and the central tendency $\theta(t)$, as well as the distance between $\theta(t)$ and $\bar{\theta} .{ }^{12}$ More explicitly,

$$
E^{Q}[V(T) \mid I(t)]=\bar{\theta}+\delta(\tau)[\theta(t)-\bar{\theta}]+\exp (-\kappa \tau)[V(t)-\theta(t)]
$$

where

$$
\delta(\tau)=\frac{\kappa}{\kappa-\bar{\kappa}} \exp (-\bar{\kappa} \tau)-\frac{\bar{\kappa}}{\kappa-\bar{\kappa}} \exp (-\kappa \tau) .
$$

Importantly, the CSQR model introduces stochastic volatility that is not spanned by the VIX, since the conditional variance under this model is an affine function of $V(t)$ and $\theta(t)$ (see Appendix B).

In turn, we add three empirically relevant features to the LOU model: a time varying mean, jumps and stochastic volatility. We consider these extensions first in isolation, and then in combination. As a general rule, we model the price of risk of the continuous part of the diffusions as in the one-factor models, while we assume that jump risk is not priced. All in all, we compare the following cases:

- Central tendency (CTOU):

$$
d \log V(t)=\kappa[\theta(t)-\log V(t)] d t+\sigma d W_{v}^{Q}(t),
$$

\footnotetext{
${ }^{12}$ Unlike in the SQR model, the convergence of (10) to the long run mean is not necessarily a monotonic function of $\tau$.
} 
with

$$
d \theta(t)=\bar{\kappa}[\bar{\theta}-\theta(t)] d t+\bar{\sigma} d W_{\theta}^{Q}(t)
$$

where $W_{v}^{Q}(t)$ and $W_{\theta}^{Q}(t)$ are independent Brownian motions. We specify the prices of risk that link the Wiener processes under $\mathrm{P}$ and $\mathrm{Q}$ as

$$
\begin{aligned}
& d W_{v}^{Q}(t)=d W_{v}^{P}(t)+\varsigma d t \\
& d W_{\theta}^{Q}(t)=d W_{\theta}^{P}(t)+\bar{\varsigma} d t .
\end{aligned}
$$

These specifications ensure that the process followed by the log VIX is also affine under the real measure.

We show in Appendix $\mathrm{C}$ that the exact discretisation of $\log V(t)$ in the above model is a Gaussian $\operatorname{ARMA}(2,1)$ process, which is consistent with the evidence reported in Section 2 (see Figure 2). Therefore, its likelihood function can be computed in closed form. As discussed in Jegadeesh and Pennacchi (1996) and Balduzzi, Das, and Foresi (1998), (12) allows the volatility index to revert towards a time varying central tendency whose long run mean is $\bar{\theta}$. As a consequence, the conditional mean of $\log V(T)$ is a function of the distance between $\log V(t)$ and the central tendency $\theta(t)$, as well as the distance between $\theta(t)$ and $\bar{\theta} \cdot{ }^{13}$ More explicitly,

$$
\begin{aligned}
E^{Q}[\log V(T) \mid I(t)]= & \bar{\theta}+\delta(\tau)[\theta(t)-\bar{\theta}] \\
& +\exp (-\kappa \tau)[\log V(t)-\theta(t)],
\end{aligned}
$$

where $\delta(\tau)$ is given by (11). Notice that, although (10) and (15) have a similar structure, the two formulas are not equivalent because the VIX appears in levels in (10). In addition, the CTOU model can equivalently be expressed as the "superposition" (i.e. sum) of two LOU factors. Therefore, the structure in (12) is not restrictive for the CTOU. ${ }^{14}$

- Jumps (LOUJ):

$$
d \log V(t)=\kappa[\bar{\theta}-\log V(t)] d t+\sigma d W_{v}^{Q}(t)+d Z(t)-\frac{\lambda}{\kappa \delta} d t
$$

\footnotetext{
${ }^{13}$ As in the CSQR model, the convergence of (15) to the long run mean is not necessarily a monotonic function of $\tau$.

${ }^{14}$ The "component" GARCH model proposed by Ding and Granger (1996) to capture "long memory" features of volatility (see also Engle and Lee, 1999) can be regarded as a discrete time analogue to (12).
} 
where $Z(t)$ is a pure jump process independent of $W_{v}^{Q}(t)$, with intensity $\lambda$, and whose jump amplitudes are exponentially distributed with mean $1 / \delta$, or $\operatorname{Exp}(\delta)$ for short. Note that the last term in (16) simply introduces a constant shift in the distribution of $\log V(t)$ which ensures that $\bar{\theta}$ remains the long-run mean of $\log V(t)$. Jumps in models for instantaneous volatility have been previously considered by Duffie, Pan, and Singleton (2000) and Eraker, Johannes, and Polson (2003), among others, while Todorov and Tauchen (2011) also consider jumps in modelling the VIX. Unlike pure diffusions, this model allows for sudden movements in volatility indices, which nevertheless have lasting effects due to the fact that the meanreversion parameter $\kappa$ is bounded. Again, we assume (13) for the price of diffusion risk.

- Stochastic volatility (LOUSV):

$$
d \log V(t)=\kappa[\bar{\theta}-\log V(t)] d t+\sqrt{\omega(t)} d W_{v}^{Q}(t)
$$

where $\omega(t)$ follows an OU- $\Gamma$ process, which belongs to the class of Lévy OU processes considered by Barndorff-Nielsen and Shephard (2001). Specifically,

$$
d \omega(t)=-\bar{\lambda} \omega(t) d t+d \bar{Z}(t)
$$

where $\bar{Z}(t)$ is a pure jump process with intensity $\bar{\lambda}$ and $\operatorname{Exp}(\bar{\delta})$ jump amplitude, while $W_{v}^{Q}(t)$ is an independent Brownian motion. We use this extension to assess to what extent the price distortions in the previous models are due to the assumption of constant volatility over time. Importantly, the model that we adopt is consistent with the presence of mean reversion in $\omega(t)$, since

$$
\left.E^{Q}[\omega(T)) \mid \omega(t)\right]=\bar{\delta}^{-1}+\exp (-\bar{\lambda} \tau)\left[\omega(t)-\bar{\delta}^{-1}\right]
$$

Hence, $\bar{\delta}^{-1}$ can be interpreted as the long run mean of the instantaneous volatility of the $\log$ VIX, while $\bar{\lambda}$ will be the corresponding mean reversion parameter. Another non-trivial advantage of this model over other alternatives such as a square root process for $\omega(t)$ is that it allows the valuation of derivatives by inverting the conditional characteristic function (see Appendix $\mathrm{C}$ for details). Once again, we consider a price of diffusion risk proportional to instantaneous volatility to ensure 
that the log VIX under the real measure remains an affine process (see Dai and Singleton, 2000). Specifically, we assume

$$
d W_{v}^{Q}(t)=d W_{v}^{P}(t)+\varsigma_{\omega} \sqrt{\omega(t)} d t .
$$

Under this specification, the price of risk has a stronger impact when the volatility of the VIX is larger.

- Central tendency and jumps (CTOUJ):

$$
d \log V(t)=\kappa[\theta(t)-\log V(t)] d t+\sigma d W_{v}^{Q}(t)+d Z(t)-\frac{\lambda}{\kappa \delta} d t
$$

where $\theta(t)$ follows the diffusion (12) and $Z(t)$ is a pure jump process with intensity $\lambda$ and $\operatorname{Exp}(\delta)$ jump amplitude, while $W_{v}^{Q}(t)$ and $W_{\theta}^{Q}(t)$ are independent Brownian motions. We again introduce a constant shift in (20) to ensure that $\bar{\theta}$ is the long run mean of both $\theta(t)$ and $\log V(t)$. As in the CTOU model, we assume that the prices of risk are given by (13) and (14).

- Central tendency and stochastic volatility (CTOUSV):

$$
d \log V(t)=\kappa[\theta(t)-\log V(t)] d t+\sqrt{\omega(t)} d W_{v}^{Q}(t)
$$

where $\theta(t)$ follows the diffusion (12) while $\omega(t)$ is defined in (17). As in previous cases, the jump variable $\bar{Z}(t)$ and the Brownian motions are mutually independent. Similarly, we consider the prices of risk specifications (14) and (19).

Despite the apparent differences between (13), (14) and (19), they are all special cases of a generic specification. In particular, following Cheridito, Filipović, and Kimmel (2007), we can write the risk neutral probability measure in terms of the real measure for all the extensions that we consider as

$$
q=\exp \left[-\int_{t}^{T}\left(\Lambda_{v}(s) d W_{v}(s)+\Lambda_{\theta}(s) d W_{\theta}(s)\right)-\frac{1}{2} \int_{t}^{T}\left(\Lambda_{v}^{2}(s)+\Lambda_{\theta}^{2}(s)\right) d s\right],
$$

where $\Lambda_{v}(t)=\varsigma_{\omega} \sqrt{\omega(t)}$ and $\Lambda_{\theta}(t)=\bar{\varsigma}$ in our case. ${ }^{15}$

Except for the CTOU model, it is not generally possible to price derivative contracts for these extensions in closed form. However, it is possible to obtain the required prices

\footnotetext{
${ }^{15}$ Note that $\omega(t)=\sigma^{2}$ in the extensions without stochastic volatility. Accordingly, we define $\varsigma=\varsigma_{\omega} \sigma$ in those cases.
} 
by Fourier inversion of the conditional characteristic function. In particular, we use formula (5) in Carr and Madan (1999) to invert the relevant characteristic functions of the extensions to the LOU model (see Appendix C). However, this approach is not valid for the CSQR model, where we need to follow recent results by Amengual and Xiu (2012) to invert the characteristic function (see Appendix B).

Some of the previous extensions introduce as additional factors a time varying tendency, a time varying volatility or both, which we need to filter out for estimation purposes. In this regard, we use the standard Kalman filter to "estimate" $\theta(t)$. And although the jump variable $Z(t)$ in (16) can also be interpreted as an additional factor, its impact cannot be separately identified from the impact of the diffusion shocks $d W_{v}^{Q}(t)$ because both variables share the same mean reversion coefficient $\kappa$. Finally, following Trolle and Schwartz (2009) and others, we employ the extended Kalman filter to deal with $\omega(t)$ (see once again Appendix C for further details).

\subsection{Empirical performance}

Table 3 reports the in- and out-of-sample RMSE's of the extensions introduced in the previous section. As expected, the CSQR process is able to yield much smaller RMSE's than the SQR and LOU models, both in and out-of-sample. Nevertheless, the CTOU model achieves a slightly superior fit, especially out-of-sample. In any case, a persistent time-varying mean seems to capture a crucial feature of the data. On the other hand, the introduction of jumps in the LOU model does not introduce improvements in the aggregate RMSE's. Similarly, adding jumps to the CTOU model does not substantially improve the fit either. However, it is important to emphasise that Table 3 does not assess the importance of jumps on the historical dynamics of the VIX, only their pricing implications.

In contrast, we find significant improvements when we consider stochastic volatility. The LOUSV introduces important reductions in the RMSE's, but the combination of central tendency and stochastic volatility provided by the CTOUSV model yields the overall best fit. Importantly, this model is able to describe the out-of-sample behaviour of the VIX during the more extreme periods of the financial crisis.

Figure 4 compares the empirical cdf's of the square pricing errors of futures and option prices separately. This figure shows that the CTOUSV model dominates in the first order 
stochastic sense all the other models for both futures and options. The ordering of the remaining models, though, depends on the type of derivative asset considered. In the case of futures, the CTOUSV is closely followed by the CSQR and then by the other central tendency specifications (CTOU and CTOUJ), which display almost identical results. In turn, they are followed by the LOUSV, LOUJ, LOU, and SQR models. But for options, the second best model is LOUSV, which is followed by the CTOUJ, LOUJ, CTOU, LOU, SQR and CSQR models. Thus, we observe that the central tendency is relatively more important for futures, while stochastic volatility offers greater gains on options. At the same time, a central tendency does not harm the option pricing performance of the CTOUSV model, while stochastic volatility does not cause any distortions to futures prices. This is not surprising, given that we show in Appendix $\mathrm{C}$ that option prices for the extensions of the LOU model do not depend on $\theta(t)$ once we condition on the current futures price. As for jumps, Figure 4 shows that they do indeed help in pricing options but they hardly provide any improvement for futures. The small impact of jumps on futures is again to be expected because we can also show that jumps in the LOUJ model generate identical futures prices as a LOU model up to first order. ${ }^{16}$ And, compared to stochastic volatility, jumps seem to yield a minor improvement even for options. In this sense, Bakshi, Cao, and Chen (1997) find that, once stochastic volatility is modelled, adding jumps only leads to second-order pricing improvements. Lastly, the SQR model offers the worst overall results, while the CSQR model also yields a poor fit for option prices.

Figure 5a compares the "actual" futures prices for a constant 30-day maturity, which we obtain by interpolation of the adjacent contracts, with the daily estimates of 30day futures prices generated by the SQR, LOU and CTOU models. By focusing on a constant maturity, we can not only compare the absolute magnitude of the pricing errors, but also their sign and persistence. As can be seen, the pricing errors of the SQR process are larger than those of the LOU process, especially after mid 2007. In turn, those of the LOU model are not only substantially larger than those of the CTOU, but they also display much stronger persistence. For instance, the SQR and LOU models

\footnotetext{
${ }^{16}$ Formally, when we consider a Taylor expansion of the futures price formula of model LOUJ around $\lambda / \delta$ for $\lambda / \delta>0$ but small, we only observe deviations from the LOU expression for the second and higher terms. This probably reflects the fact that the conditional mean of the VIX is essentially unaffected by the presence of jumps although they alter skewness and kurtosis.
} 
systematically underprice futures from October 2008 until the end of the sample. The slower mean reverting properties of the VIX are probably responsible for these persistent biases in the one-factor models. We can also observe in Figure $5 \mathrm{~b}$ that the pricing errors of the CSQR, CTOU and CTOUSV models are almost identical, albeit with slightly smaller oscillations for the CTOUSV model. This feature confirms once again that central tendency is the most relevant extension for pricing futures.

Table 4 reports the parameter estimates that we obtain under the real measure in the extensions to the SQR and LOU models we are considering. In the models with central tendency we observe fast mean reversion of the VIX to $\theta(t)$, which in turn mean-reverts rather more slowly to its long rung mean $\bar{\theta}$ (i.e. $\kappa \gg \bar{\kappa}$ ). Importantly, the estimates of these parameters are very stable in models with central tendency. In contrast, jump intensities vary substantially depending on the sample period considered for estimation. Specifically, we obtain smaller values of $\lambda$ when we include the crisis period. For the full sample, we estimate around 5 jumps per year in the LOUJ specification, and almost 7 jumps per year in the CTOUJ model. On the other hand, the estimates of the mean reversion parameter $\bar{\lambda}$ in the stochastic volatility models tend to be larger when central tendency is not simultaneously included. Since this parameter is also responsible for jump intensity in the OU- $\Gamma$ model, this result has two interesting implications. First, a smaller value of $\bar{\lambda}$ tends to reduce jump activity in $\bar{Z}(t)$. Specifically, the expected number of jumps per year decreases from 15 in the LOUSV model to 2 in the CTOUSV extension. Second, the deviations of $\omega(t)$ from its long run mean are more persistent in the CTOUSV case because mean reversion is slower the smaller $\bar{\lambda}$ is, as (18) indicates. These features are also important from a time series perspective. As mentioned before, central tendency is consistent with $\operatorname{ARMA}(2,1)$ dynamics in discrete time, while stochastic volatility introduces GARCH-type persistent variances for the log VIX.

\subsection{Risk pricing}

Table 5 shows the point estimates of the price of risk parameters for our preferred model. We also conduct Wald tests to assess the statistical significance of these estimates. The price of risk related to the VIX equation (19), $\varsigma_{\omega}$, is highly significant in the two samples. In contrast, $\bar{\varsigma}$, which is the price of risk related to the innovations in (12), is insignificant in both cases. 
The negative sign of $\varsigma_{\omega}$ implies a more adverse distribution in the $Q$ measure, because the VIX mean reverts towards a higher long run level than in the $P$ measure. This result, which is consistent with the evidence found by Egloff, Leippold, and Wu (2010), should be taken into account in inferring the market expectation about future values of the VIX from its derivatives.

The difference between the properties of the VIX under the real and risk-neutral measures implies, within the framework of our model, that an economically important systematic risk is priced in the market of VIX derivatives. In addition, the statistical significance of the estimate of $\varsigma_{\omega}$ implies that the impact of the price of risk is larger when the VIX is more volatile (see equation 19).

\subsection{Evolution of the factors}

Figure 6 a shows that the filtered values of the central tendency factor $\theta(t)$ are rather insensitive to the particular specification that we use. We have also found that our preferred CTOUSV model generates almost indistinguishable filtered values for $\theta(t)$ for the two estimation samples that we consider. Not surprisingly, Figure 6a also confirms that the log of the VIX oscillates around $\theta(t)$, which in turn changes over time rather more slowly. In fact, the main reason for central tendency models to work so well during the recent financial crisis is because they allow for large temporal deviations of $\theta(t)$ from

its long term value $\bar{\theta}$, thereby reconciling the large increases of the VIX observed during that period with mean reversion over the long term.

Figure $6 \mathrm{~b}$ compares the filtered instantaneous volatilities of the LOUSV and CTOUSV models. Interestingly, both series display an almost identical pattern, with substantial persistent oscillations over time. This figure also shows that large increases in the value of the VIX are associated to volatile periods, as measured by $\omega(t)$. This is particularly visible in August 2007, October 2008 and May 2010. As with central tendency, we have found that the filtered values of $\omega(t)$ obtained from the CTOUSV model are also quite insensitive to the sample period used to estimate the model parameters.

We compute the 30-day ahead standard deviations of $\log V(t)$ implied by the CTOUSV model to assess the extent to which the filtered values of $\omega(t)$ make sense. In Figure 6c we compare those standard deviations with the Black (1976) implied volatilities of at-the-money options that are exactly 30 days from expiration, which we again obtain 
by interpolation. The high correlation between the two series shows that the filtered values of stochastic volatility are indeed related to the changing perceptions of the market about the standard deviation of the VIX.

Finally, Figure 6d compares the one-day-ahead standard deviations under the real measure implied by the models with the standard deviations obtained from a discrete $\operatorname{ARMA}(2,1)-\operatorname{GARCH}(1,1)$ model for the log VIX. We only consider the two most relevant extensions to the LOU model to avoid cluttering the picture. As we have already mentioned in Section 4, both the SQR and LOU models yield time varying variances for the VIX. However, the variance implied by the SQR process seems to be too high until mid 2007 and too low afterwards. The LOU model performs better over tranquil periods, but it underestimates actual volatility levels in the most severe phases of the financial crisis. In contrast, the CTOUJ and especially the CTOUSV do a much better job, while the CSQR significantly overestimates the standard deviations during low volatility periods.

\subsection{Term structures of derivatives and implied volatility skews}

So far, we have focused on the overall empirical performance of the different models. In principle, though, our results could change for different time horizons or different degrees of moneyness. For that reason, Table 6 shows the RMSE's of futures contracts for different ranges of maturity. We observe that the models without central tendency tend to yield larger distortions for longer maturities. In contrast, the models with central tendency are relatively worse at pricing the shortest maturity. Nevertheless, even the worst RMSE of the CTOUSV model is still much smaller than the best RMSE of either the SQR or the LOU models without central tendency.

To gain some additional insight, in Figure 7 we look at the term structure of futures prices for four particularly relevant days. Futures prices were extremely low on June 21, 2007, even though the first warning signals about the impending crisis were starting to appear. Prices had already risen significantly by August 15, 2008, one month before the collapse of Lehman Brothers. Nevertheless, they were much higher on November 20, 2008, which is the day in which the VIX reached its maximum historical closing value at 80.86. The increase is particularly remarkable at the short end of the curve. Since then, though, VIX futures prices significantly came down until the beginning of April 2010, right before the European sovereign debt crisis. The figure confirms that a central 
tendency is crucial for the purposes of reproducing the changes in the level and slope of the actual term structure of VIX futures prices. We can also observe that the LOU and LOUJ processes yield futures prices which are almost identical, while the CTOUJ model can be barely distinguished from the CTOU extension.

Tables 7 and 8 provide the RMSE's of calls and puts for different ranges of maturity and moneyness. In this case, we generally observe the highest price distortions for atthe-money call and put options. The SQR, CSQR, LOU, LOUJ, CTOU and CTOUJ models seem to do a better job for moneyness smaller than -0.3 . However, once again we can confirm that stochastic volatility models provide the best fit uniformly across all moneyness and maturity ranges.

In Figure 8 we assess the ability of the different models to fit the average implied volatility skews in Figures $3 \mathrm{~b}$ and 3c. Once again, we only plot the most relevant extensions to the LOU model to avoid cluttering the pictures. In addition to the average implied skews of actual prices, we also consider $5 \%$ and $95 \%$ percentiles as a measure of dispersion. The figures confirm that the LOU model yields a constant implied volatility for all strikes. In contrast, the SQR and CSQR yield volatility skews but with a negative slope, which is inconsistent with the positive slope in the data. In contrast, both the CTOUJ and CTOUSV models are able to reproduce this positive slope, with the latter generally providing the best fit. Specifically, the CTOUJ model does not capture the shifts of the implied skews due to rises in volatility and it also performs poorly for low strikes.

\section{Conclusions}

We carry out an extensive empirical analysis of VIX derivatives valuation models. We consider daily prices of futures and European options from February 2006 until December 2010. Therefore we not only cover an unusually tranquil period, but also the early turbulences that took place between August 2007 and August 2008, the worst months of the recent financial crisis (autumn 2008), as well as the months in 2010 in which the European sovereign debt crisis unfolded. These markedly different periods provide a very useful testing ground to assess the empirical performance of the different pricing models. We estimate the models using not only futures and options data, but also historical data on the VIX itself, which allows us to look at the relationship between 
real and risk neutral measures. We initially focus on the two existing mean-reversion models: the square root (SQR) and the log-normal Ornstein-Uhlenbeck processes (LOU). Although SQR is more popular in the empirical literature, we find that the LOU model yields a better fit, especially during the crisis. However, both models yield large price distortions during the crisis. In addition, they do not seem to capture either the level or the slope of the term structure of futures prices, or indeed the volatility skews. Part of the problem is that these models implicitly assume that volatility either mean reverts at a simple exponential rate or does not mean revert at all, which cannot accommodate the long and persistent swings of the VIX observed in our sample. In this sense, we show that the simple $\mathrm{AR}(1)$ structure that they imply in discrete time is not consistent with the empirical evidence of ARMA dynamics for the VIX. In addition, these models are also inconsistent with the strong presence of GARCH-type heteroskedasticity that we find in this volatility index.

We investigate the potential sources of mispricing by considering several empirically relevant generalisations. In particular, we consider the concatenated SQR process (CSQR), which substantially extends the SQR model by introducing a time varying central tendency and allowing for unspanned stochastic volatility. We also extend the LOU model by introducing a time varying central tendency, jumps and stochastic volatility. Our parameter estimates indicate that the VIX rapidly mean-reverts to a central tendency, which in turn reverts more slowly to a long run constant mean. This flexible structure can reconcile the large variations of the VIX over our sample period with meanreversion to a long run constant value. Except for the CTOU model, though, it is not generally possible to price derivatives in closed form for the extensions that we consider. For that reason, we obtain the required prices by Fourier inversion of the conditional characteristic function.

Interestingly, our results indicate that a time varying central tendency is crucial for pricing futures, regardless of whether the model is expressed in levels or logs. We also find evidence of time varying volatility in the VIX. As expected, stochastic volatility plays a much more important role for options while leaving futures prices almost unaffected. The CSQR model, though, seems unable to generate the positive slope of the option implied volatility skews. It is also worth mentioning that jumps only provide a minor improvement for options and do not change futures prices (up to first order). 
Nevertheless, we would like to emphasise that jumps seem to be a relevant feature to describe the historical dynamics of the VIX, even though they only yield second order gains for pricing VIX derivatives.

Importantly, our results remain valid when we focus exclusively on the out-of-sample performance with parameters estimated using data prior to the autumn of 2008. In view of these findings, we conclude that a generalised LOU model that combines a time varying central tendency with stochastic volatility is needed to obtain a good pricing performance during bull and bear markets, as well as to capture the term structures of VIX futures and options, and the positive slope of the implied volatility skews of options. Interestingly, the price of risk of this specification is highly significant and implies that the VIX mean reverts towards a higher long run mean under the risk neutral measure than under the real measure. The difference between the properties of the VIX under the real and risk-neutral measures implies that an economically important systematic risk is priced in the market of VIX derivatives.

Given the relationship between the observed VIX index and the unobserved integrated volatility of the S\&P500, our analysis also has important implications for the models and stationarity of this broad stock index commonly used by participants in markets for stock index options. Specifically, our results imply that stochastic volatility models for the S\&P500 should allow for slow mean reversion by including two volatility factors, and a time-varying volatility of volatility. Amengual (2009) considers such a model for volatility swaps.

We could extend our empirical exercise to other recently introduced volatility derivatives such as binary options or American options on VIX futures, or even the futures and options on the CBOE Gold ETF Volatility Index. Sophisticated filtering procedures for the volatility of VIX might also be worth exploring, as well as tractable ways of modelling jump and stochastic volatility risk for Lévy processes. It would also be interesting to investigate the incremental information content of the contemporaneous observations of the S\&P500 over and above the spot VIX for pricing VIX futures, and above and beyond VIX futures for pricing VIX options. This question is also relevant for the purposes of integrating the valuation of VIX derivatives with the valuation of the underlying options on the S\&P500 that are used to compute this volatility index, as suggested by Lin and Chang (2009). We plan to address these points in subsequent research. 


\section{References}

Amengual, D. (2009). The term structure of variance risk premia. Mimeo Princeton University.

Amengual, D. and D. Xiu (2012). Delving into risk premia: reconciling evidence from the S\&P 500 and VIX derivatives. Work in progress.

Andersen, T. G., T. Bollerslev, and F. X. Diebold (2009). Parametric and nonparametric volatility measurement. In Y. Aït-Sahalia and L. P. Hansen (Eds.), Handbook of Financial Econometrics, Volume 1, pp. 67-138. North-Holland Press.

Andersen, T. G. and O. Bondarenko (2007). Construction and interpretation of modelfree implied volatility. NBER Working Paper No. W13449.

Bakshi, G., C. Cao, and Z. Chen (1997). Empirical performance of alternative option pricing models. Journal of Finance 52, 2003-2049.

Balduzzi, P., S. R. Das, and S. Foresi (1998). The central tendency: a second factor in bond yields. Review of Economics and Statistics 80, 62-72.

Barndorff-Nielsen, O. and N. Shephard (2001). Non-Gaussian Ornstein-Uhlenbeck-based models and some of their uses in financial economics. Journal of the Royal Statistical Society. Series B (Statistical Methodology) 63, 167-241.

Bates, D. S. (2000). Post-'87 crash fears in the S\&P 500 futures option market. Journal of Econometrics 94, 181-238.

Bates, D. S. (2012). U.S. stock market crash risk, 1926-2010. Journal of Financial Economics 105, 229-259.

Black, F. (1976). The pricing of commodity contracts. Journal of Financial Economics 3, 167-179.

Carr, P. and D. B. Madan (1999). Option valuation using the fast Fourier transform. Journal of Computational Finance 2, 61-73.

Carr, P. and L. Wu (2006). A tale of two indices. Journal of Derivatives 13, 13-29.

CBOE (2009). The CBOE volatility index - VIX. White Paper (available at www.cboe.com/micro/vix).

Cheridito, P., D. Filipović, and R. L. Kimmel (2007). Market price of risk specifications for affine models: theory and evidence. Journal of Financial Economics 83, 123-170.

Cox, J. C., J. E. Ingersoll, and S. A. Ross (1985). A theory of the term structure of interest rates. Econometrica 53, 385-407.

Dai, Q. and K. J. Singleton (2000). Specification analysis of affine term structure models. Journal of Finance 55, 1943-1978. 
Demos, A. and E. Sentana (1998). Testing for GARCH effects: a one-sided approach. Journal of Econometrics 86, 97-127.

Detemple, J. and C. Osakwe (2000). The valuation of volatility options. European Finance Review 4, 21-50.

Ding, Z. and C. W. J. Granger (1996). Modeling volatility persistence of speculative returns: a new approach. Journal of Econometrics 73, 185-215.

Dotsis, G., D. Psychoyios, and G. Skiadopoulos (2007). An empirical comparison of continuous-time models of implied volatility indices. Journal of Banking 85 Finance 31, $3584-3603$

Duffie, D., J. Pan, and K. Singleton (2000). Transform analysis and asset pricing for affine jump-diffusions. Econometrica 68, 1343-1376.

Dumas, B., J. Fleming, and E. Whaley (1998). Implied volatility functions: empirical tests. Journal of Finance 53, 2059-2106.

Egloff, D., M. Leippold, and L. Wu (2010). The term structure of variance swap rates and optimal variance swap investments. Journal of Financial and Quantitative Analysis 45, 1279-1310.

Engle, R. F. and G. J. Lee (1999). A permanent and transitory component model of stock return volatility. In R. F. Engle and H. White (Eds.), Cointegration, causality, and forecasting: a festschrift in honor of Clive W.J. Granger, pp. 475-497. Oxford University Press.

Eraker, B., M. Johannes, and N. Polson (2003). The impact of jumps in volatility and returns. Journal of Finance 53, 1269-1300.

Fackler, P. L. (2000). Moments of affine diffusions. North Carolina State University Working Paper.

French, K. R., G. W. Schwert, and R. F. Stambaugh (1987). Expected stock returns and volatility. Journal of Financial Economics 19, 3-29.

Grünbichler, A. and F. A. Longstaff (1996). Valuing futures and options on volatility. Journal of Banking \& Finance 20, 985-1001.

Hansen, L. P. and J. A. Scheinkman (2009). Long-term risk: an operator approach. Econometrica 7\%, 177-234.

Heston, S. L. (1993). A closed-form solution for options with stochastic volatility with applications to bond and currency options. Review of Financial Studies 6, 327-343. 
Jegadeesh, N. and G. G. Pennacchi (1996). The behavior of interest rates implied by the term structure of eurodollar futures. Journal of Money, Credit and Banking 28, 426-446.

León, A. and E. Sentana (1997). Pricing options on assets with predictable white noise returns. LSE FMG Discussion Papers 267.

Lin, Y. N. and C. H. Chang (2009). VIX option pricing. Journal of Futures Markets 29, $523-543$.

Pagan, A. R. and G. W. Schwert (1990). Alternative models for conditional stock volatility. Journal of Econometrics 45, 267-290.

Rhoads, R. (2011). Trading VIX derivatives: trading and hedging strategies using VIX futures, options and exchange traded notes. New York: John Wiley and Sons.

RiskMetrics Group (1996). Riskmetrics Technichal document.

Schwert, G. W. (1990). Stock returns and real activity: a century of evidence. Journal of Finance 45, 1237-1257.

Schwert, G. W. (2011). Stock volatility during the recent financial crisis. European Financial Management 17, 789-805.

Sepp, A. (2008). VIX option pricing in a jump-diffusion model. Risk Magazine, 84-89. April.

Song, Z. and D. Xiu (2012). A tale of two option markets: state-price densities implied from S\&P500 and VIX option prices. Mimeo Chicago Booth.

Szado, E. (2009). VIX futures and options - A case study of portfolio diversification during the 2008 financial crisis. Journal of Alternative Investments 12, 68-85.

Todorov, V. and G. Tauchen (2011). Volatility jumps. Journal of Business and Economic Statistics 29, 356-371.

Trolle, A. B. and E. S. Schwartz (2009). Unspanned stochastic volatility and the pricing of commodity derivatives. Review of Financial Studies 22, 4423-4461.

Wang, Z. and R. T. Daigler (2011). The performance of VIX option pricing models: empirical evidence beyond simulation. Journal of Futures Markets 31, 251-281.

Whaley, R. E. (1993). Derivatives on market volatility: hedging tools long overdue. Journal of Derivatives 1, 71-84.

Zhang, J. E. and Y. Zhu (2006). VIX futures. Journal of Futures Markets 26, 521-531. 


\section{A Affine models conditional characteristic function}

The extensions that we consider belong to the class of affine jump-diffusion state processes analysed by Duffie, Pan, and Singleton (2000). In particular, consider an $N$-dimensional vector $\mathbf{Y}(t)$ that satisfies the diffusion

$$
d \mathbf{Y}(t)=\mathbf{K}(\boldsymbol{\Theta}-\mathbf{Y}(t)) d t+\sqrt{\mathbf{S}(t)} d \mathbf{W}(t)+d \mathbf{Z}(t)
$$

where $\mathbf{W}(t)$ is an $N$-dimensional vector of independent standard Brownian motions, $\mathbf{K}$ is an $N \times N$ matrix, $\Theta$ is a vector of dimension $N, \mathbf{S}(t)$ is a diagonal matrix of dimension $N$ whose $i^{t h}$ diagonal element is $c_{i 0}+\mathbf{c}_{i 1}^{\prime} \mathbf{Y}(t)$, and finally, $\mathbf{Z}(t)$ is a multivariate pure jump process with intensity $\lambda$ whose jump amplitudes have joint density $f_{J}(\cdot)$.

Duffie, Pan, and Singleton (2000) show that the conditional characteristic function of $\mathbf{Y}(T)$ can be expressed as

$$
\begin{aligned}
\phi_{Y}(t, T, \mathbf{u}) & =E\left[\exp \left(i \mathbf{u}^{\prime} \mathbf{Y}(T)\right) \mid I(t)\right] \\
& =\exp \left(\varphi_{0}(\tau)+\varphi_{Y}^{\prime}(\tau) \mathbf{Y}(t)\right),
\end{aligned}
$$

where $\varphi_{0}(\tau)$ and $\varphi_{Y}(\tau)$ satisfy the following system of differential equations:

$$
\begin{gathered}
\dot{\boldsymbol{\varphi}}_{Y}(\tau)=-\mathbf{K}^{\prime} \boldsymbol{\varphi}_{Y}(\tau)+\frac{1}{2} \boldsymbol{\varsigma}_{Y}(\tau), \\
\dot{\varphi}_{0}(\tau)=\boldsymbol{\Theta}^{\prime} \mathbf{K} \boldsymbol{\varphi}_{Y}(\tau)+\frac{1}{2} \boldsymbol{\varphi}_{Y}^{\prime}(\tau) \operatorname{diag}\left(\mathbf{c}_{0}\right) \boldsymbol{\varphi}_{Y}(\tau)+\lambda\left[J\left(\boldsymbol{\varphi}_{Y}(\tau)\right)-1\right],
\end{gathered}
$$

where $J(\mathbf{u})=\int \exp \left(\mathbf{u}^{\prime} \mathbf{x}\right) f_{J}(\mathbf{x}) d \mathbf{x}$ and $\boldsymbol{\varsigma}_{Y}(\tau)$ is an $N$-dimensional vector whose $k^{\text {th }}$ element is $\varsigma_{Y, k}(\tau)=\varphi_{Y}^{\prime}(\tau) \operatorname{diag}\left(\mathbf{c}_{k 1}\right) \boldsymbol{\varphi}_{Y}(\tau)$.

\section{B The CSQR model}

Given the prices of risk (8) and (9), we can write the CSQR process under the real measure as

$$
d V(t)=\kappa^{P}\left[\theta^{P}(t)-V(t)\right] d t+\sigma \sqrt{V(t)} d W_{v}^{P}(t)
$$

with

$$
d \theta^{P}(t)=\bar{\kappa}^{P}\left[\bar{\theta}^{P}-\theta^{P}(t)\right] d t+\bar{\sigma}^{P} \sqrt{\theta(t)} d W_{\theta}^{P}(t),
$$

where $\kappa=\kappa^{P}+\sigma \varsigma, \bar{\kappa}=\bar{\kappa}^{P}+\bar{\sigma} \bar{\varsigma}$,

$$
\begin{aligned}
\bar{\theta} & =\frac{\kappa^{P} \bar{\kappa}^{P}}{\left(\kappa^{P}+\sigma \varsigma\right)\left(\bar{\kappa}^{P}+\bar{\sigma} \bar{\varsigma}\right)} \bar{\theta}^{P}, \\
\bar{\sigma} & =\bar{\sigma}^{P} \sqrt{\frac{\kappa^{P}}{\kappa^{P}+\sigma \varsigma}},
\end{aligned}
$$


and

$$
\theta(t)=\frac{\kappa^{P}}{\kappa^{P}+\sigma \varsigma} \theta^{P}(t)
$$

Let $\mathbf{X}(t)=[\theta(t), V(t)]^{\prime}$. Following Fackler (2000), it can be shown that the conditional mean and variance of $X(T)$ given information known at time $t$ is the affine function of $\mathbf{X}(t)$ :

$$
\left[\begin{array}{c}
E[X(T) \mid I(t)] \\
\operatorname{vec}[V[X(T) \mid I(t)]]
\end{array}\right]=\left[\begin{array}{c}
\mathbf{m}_{0} \\
\mathbf{v}_{0}
\end{array}\right]+\left[\begin{array}{c}
\mathbf{M}_{1} \\
\mathbf{V}_{1}
\end{array}\right] \mathbf{X}(t),
$$

where $\mathbf{m}_{0}, \mathbf{v}_{0}, \mathbf{M}_{1}$ and $\mathbf{V}_{1}$ are $2 \times 1,4 \times 1,2 \times 2$ and $4 \times 2$ vectors and matrices, respectively, such that

$$
\begin{aligned}
{\left[\begin{array}{c}
\mathbf{m}_{0} \\
\mathbf{v}_{0}
\end{array}\right] } & =\left[\exp (\tau \mathbf{A})-\mathbf{I}_{6}\right] \mathbf{A}^{-1} \mathbf{a}, \\
{\left[\begin{array}{c}
\mathbf{M}_{1} \\
\mathbf{V}_{1}
\end{array}\right] } & =\exp (\tau \mathbf{A})\left[\begin{array}{c}
\mathbf{I}_{2} \\
\mathbf{0}_{4 \times 2}
\end{array}\right],
\end{aligned}
$$

a is a $6 \times 1$ vector whose first element is $\bar{\kappa} \bar{\theta}$ and all the other elements are zero; $\mathbf{I}_{6}$ is the identity matrix of order $6 ; \mathbf{0}_{4 \times 2}$ is a $4 \times 2$ matrix of zeros; and

$$
\mathbf{A}=\left[\begin{array}{cc}
\mathbf{R} & \mathbf{0}_{2 \times 4} \\
\mathbf{\Sigma} & \left(\mathbf{R} \otimes \mathbf{I}_{2}\right)+\left(\mathbf{I}_{2} \otimes \mathbf{R}\right)
\end{array}\right]
$$

with

$$
\begin{aligned}
& \mathbf{R}=\left[\begin{array}{cc}
-\bar{\kappa} & 0 \\
\kappa & -\kappa
\end{array}\right], \\
& \boldsymbol{\Sigma}=\left[\begin{array}{cc}
\bar{\sigma}^{2} & 0 \\
0 & 0 \\
0 & 0 \\
0 & \sigma^{2}
\end{array}\right] .
\end{aligned}
$$

It can be tediously shown that $\mathbf{m}_{0}+\mathbf{M}_{1} \mathbf{X}(t)$ yields (10).

Using the results form Appendix A, we can express the conditional characteristic function of this model as

$$
\begin{aligned}
\phi_{C S Q R}(\tau, u) & =E[\exp ((\alpha+i u) V(T)) \mid I(t)] \\
& =\exp \left[\varphi_{C S Q R, 0}(\tau)+\varphi_{C S Q R, \theta}(\tau) \theta(t)+\varphi_{C S Q R, V}(\tau) V(t)\right]
\end{aligned}
$$

where

$$
\begin{aligned}
\dot{\varphi}_{C S Q R, \theta}(\tau) & =-\bar{\kappa} \varphi_{C S Q R, \theta}(\tau)+\kappa \varphi_{C S Q R, V}(\tau)+\frac{1}{2} \bar{\sigma}^{2} \varphi_{C S Q R, \theta}^{2}(\tau), \\
\dot{\varphi}_{C S Q R, V}(\tau) & =-\kappa \varphi_{C S Q R, V}(\tau)+\frac{1}{2} \sigma^{2} \varphi_{C S Q R, V}^{2}(\tau), \\
\dot{\varphi}_{C S Q R, 0}(\tau) & =\bar{\kappa} \bar{\theta} \varphi_{C S Q R, \theta}(\tau) .
\end{aligned}
$$


with the conditions $\varphi_{C S Q R, \theta}(0)=0, \varphi_{C S Q R, V}(0)=\alpha+i u$, and $\varphi_{C S Q R, 0}(0)=0$. Furthermore, it can be shown that

$$
\varphi_{C S Q R, V}(t)=\frac{(\alpha+i u) \exp (-\kappa \tau)}{1-(\alpha+i u) \frac{\sigma^{2}(1-\exp (-\kappa \tau))}{2 \kappa}} .
$$

Then, we can follow Amengual and Xiu (2012) in showing that the price of a European call option with strike $K$ can be expressed as

$$
c(t, T, K)=\frac{\exp (-r \tau)}{\pi} \int_{0}^{\infty} \operatorname{Re}\left[\phi_{C S Q R}(\tau, u) \frac{\exp [-K(\alpha+i u)]}{(\alpha+i u)^{2}}\right] d u
$$

where the smoothing parameter $\alpha$ must be such that

$$
\alpha<\frac{2 \kappa}{\sigma^{2}(1-\exp (-\kappa \tau))} .
$$

\section{Extensions of LOU processes}

\section{C.1 General case}

If we place $\log V(t)$ as the first element in of $\mathbf{Y}(t)$ in (A1), then the conditional characteristic function of $\log V(t)$ will be $\phi(t, T, u)=\phi_{Y}\left(t, T, \mathbf{u}_{0}\right)$, where $\mathbf{u}_{0}=(u, 0, \cdots, 0)^{\prime}$. Following Carr and Madan (1999), the price of a call option with strike $K$ can then be expressed as

$$
c(t, T, K)=\frac{\exp (-\alpha \log (K))}{\pi} \int_{0}^{\infty} \exp (-i u \log (K)) \psi(u) d u
$$

where

$$
\psi(u)=\frac{\exp (-r \tau) \phi(t, T, u-(1+\alpha) i)}{\alpha^{2}+\alpha-u^{2}+i(1+2 \alpha) u}
$$

and $\alpha$ is a smoothing parameter. We evaluate $(\mathrm{C} 2)$ by numerical integration. In our experience, $\alpha=1.1$ yields good results.

Given that the estimation algorithm requires the evaluation of the objective function at many different parameter values, we linearise option prices with respect to $\omega(t)$ in the models with stochastic volatility to speed up the calculations. Our procedure is analogous to the treatment of other stochastic volatility models, which are sometimes linearised to employ the Kalman filter (see e.g. Trolle and Schwartz, 2009). Specifically, we linearise call prices for day $t$ around the volatility of the previous day as follows:

$$
\begin{aligned}
c_{\text {LOUSV }}(t, T, K, \omega(t)) \approx & c_{\text {LOUSV }}\left(t, T, K, \omega\left(t-\frac{1}{360}\right)\right) \\
& +\left.\frac{\partial c_{L O U S V}(t, T, K, x)}{\partial x}\right|_{x=\omega\left(t-\frac{1}{360}\right)}\left[\omega(t)-\omega\left(t-\frac{1}{360}\right)\right] .
\end{aligned}
$$


Due to the high persistence of $\omega(t)$, its previous day value turns out to be a very good predictor, which reduces the approximation error of the above expansion. In fact, the linearisation error, expressed in terms of the RMSE's of options, is very small (below $0.25 \%$ ). In any case, we calculate the exact pricing errors once we have obtained the final parameter estimates.

\section{C.2 Central tendency}

Given the prices of risk (13) and (14), the diffusions under the real measure can be expressed as

$$
\begin{aligned}
d \log V(t) & =\kappa\left[\theta^{P}(t)-\log V(t)\right] d t+\sigma d W_{v}^{P}(t), \\
d \theta^{P}(t) & =\bar{\kappa}\left[\bar{\theta}^{P}-\theta^{P}(t)\right] d t+\bar{\sigma} d W_{\theta}^{P}(t),
\end{aligned}
$$

where

$$
\bar{\theta}^{P}=\bar{\theta}+\frac{\sigma \varsigma}{\kappa}+\frac{\bar{\sigma} \bar{\varsigma}}{\bar{\kappa}}
$$

and

$$
\theta^{P}(t)=\theta(t)+\frac{\sigma \varsigma}{\kappa}
$$

Following León and Sentana (1997), it can be shown that the conditional distribution of $\log V(T)$ given information up to time $t$ is Gaussian with mean $\mu_{C T O U}(t, \tau)$ given in (15) and variance

$$
\begin{aligned}
\varphi_{\text {CTOU }}^{2}(\tau)= & \frac{\sigma^{2}}{2 \kappa}[1-\exp (-2 \kappa \tau)] \\
& +\bar{\sigma}^{2}\left(\frac{\kappa}{\kappa-\bar{\kappa}}\right)^{2}\left[\begin{array}{c}
\frac{1-\exp (-2 \bar{\kappa} \tau)}{2 \bar{\kappa}}+\frac{1-\exp (-2 \kappa \tau)}{2 \kappa} \\
-2 \frac{1-\exp (-(\kappa+\bar{\kappa}) \tau)}{\kappa+\bar{\kappa}}
\end{array}\right] .
\end{aligned}
$$

By exploiting log-normality, we can write futures prices as

$$
F_{C T O U}(t, T, V(t), \kappa, \theta, \sigma)=\exp \left(\mu_{C T O U}(t, \tau)+0.5 \varphi_{C T O U}^{2}(\tau)\right)
$$

while call prices follow the usual Black (1976) formula with volatility $\varphi_{\text {CTOU }}(\tau)$. This confirms that the prices of options do not depend on $\theta(t)$ once we condition on the futures price.

In terms of time series dynamics, it can be shown that $\theta(t)$ and $\log V(t)$ jointly follow a Gaussian VAR(1) if sampled at equally spaced intervals. Specifically,

$$
\left(\begin{array}{c}
\theta(T) \\
\log V(T)
\end{array}\right)=\mathbf{g}_{\tau}+\mathbf{F}_{\tau}\left(\begin{array}{c}
\theta(t) \\
\log V(t)
\end{array}\right)+\boldsymbol{\varepsilon}_{\tau},
$$


where

$$
\begin{gathered}
\mathbf{g}_{\tau}=\left[\begin{array}{cc}
1-\exp (-\bar{\kappa} \tau) & \exp (-\kappa \tau))
\end{array}\right] \bar{\theta}, \\
\mathbf{F}_{\tau}=\left[\begin{array}{cc}
\exp (-\bar{\kappa} \tau) & 0 \\
\frac{\kappa}{\kappa-\bar{\kappa}}[\exp (-\bar{\kappa} \tau)-\exp (-\kappa \tau)] & \exp (-\kappa \tau)
\end{array}\right] .
\end{gathered}
$$

and $\boldsymbol{\varepsilon}_{\tau} \sim$ iid $N\left(\mathbf{0}, \boldsymbol{\Sigma}_{\tau}\right)$, where $\boldsymbol{\Sigma}_{\tau}$ is a symmetric $2 \times 2$ matrix with elements

$$
\begin{gathered}
\boldsymbol{\Sigma}_{\tau}(1,1)=\frac{\bar{\sigma}^{2}}{2 \bar{\kappa}}[1-\exp (-2 \bar{\kappa} \tau)] \\
\boldsymbol{\Sigma}_{\tau}(1,2)=\frac{\kappa \bar{\sigma}^{2}}{\kappa-\bar{\kappa}}\left[\frac{1-\exp (-2 \bar{\kappa} \tau)}{2 \bar{\kappa}}-\frac{1-\exp (-(\kappa+\bar{\kappa}) \tau)}{\kappa+\bar{\kappa}}\right]
\end{gathered}
$$

and $\boldsymbol{\Sigma}_{\tau}(2,2)=\varphi_{C T O U}^{2}(\tau)$. From here, it is straightforward to obtain the marginal process followed by $\log V(t)$, which corresponds to the following $\operatorname{ARMA}(2,1)$ model:

$$
\log V(t)=h_{0}(\tau)+h_{1}(\tau) \log V(t-\tau)+h_{2}(\tau) \log V(t-2 \tau)+u(t)+g(\tau) u(t-\tau)
$$

where $u(t), u(t-\tau), \cdots \sim$ iid $N\left(0, p^{2}(\tau)\right)$ and

$$
\begin{aligned}
& h_{0}(\tau)=\bar{\theta}\left(1-h_{1}(\tau)-h_{2}(\tau)\right) \text {, } \\
& h_{1}(\tau)=\mathbf{F}_{\tau}(1,1)+\mathbf{F}_{\tau}(2,2) \text {, } \\
& h_{2}(\tau)=-\mathbf{F}_{\tau}(1,1) \mathbf{F}_{\tau}(2,2),
\end{aligned}
$$

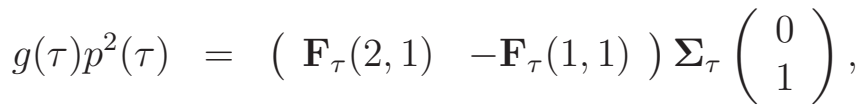

$$
\begin{aligned}
& \left(1+g^{2}(\tau)\right) p^{2}(\tau)=\left(\begin{array}{ll}
\mathbf{F}_{\tau}(2,1) & \left.-\mathbf{F}_{\tau}(1,1)\right)
\end{array}\right) \boldsymbol{\Sigma}_{\tau}\left(\begin{array}{c}
\mathbf{F}_{\tau}(2,1) \\
-\mathbf{F}_{\tau}(1,1)
\end{array}\right)+\boldsymbol{\Sigma}_{\tau}(2,2) .
\end{aligned}
$$

\section{C.3 Jumps}

Once again, we assume that $d W_{v}^{Q}(t)=d W_{v}^{P}(t)+\varsigma d t$ holds and that jump risk is not priced. Then, it can be shown that $\log V(t)$ satisfies the diffusion

$$
d \log V(t)=\kappa\left[\bar{\theta}^{P}-\log V(t)\right] d t+\sigma d W_{v}^{P}(t)+d Z(t)-\frac{\lambda}{\kappa \delta} d t
$$

under the real measure, where $\bar{\theta}^{P}=\bar{\theta}+\sigma \varsigma / \kappa$.

The conditional characteristic function reduces to

$$
\phi_{L O U J}(t, T, u)=\exp \left[\varphi_{L O U J, 0}(\tau)+\varphi_{L O U J, V}(\tau) \log V(t)\right]
$$

where

$$
\begin{aligned}
\varphi_{L O U J, 0}(\tau)= & i u\left(\bar{\theta}-\frac{\lambda}{\kappa \delta}\right)[1-\exp (-\kappa \tau)]-\frac{\sigma^{2} u^{2}}{4 \kappa}[1-\exp (-2 \kappa \tau)] \\
& +\frac{\lambda}{\kappa} \log \left[\frac{\delta-i u \exp (-\kappa \tau)}{\delta-i u}\right]
\end{aligned}
$$


and

$$
\varphi_{L O U J, V}(\tau)=i u \exp (-\kappa \tau)
$$

\section{C.4 Stochastic volatility}

We consider a price of risk such that $d W_{v}^{Q}(t)=d W_{v}^{P}(t)+\varsigma_{\omega} \sqrt{\omega(t)} d t$, but we again assume that jump risk related to $Z(t)$ is not priced. Then, the process under the real measure can be expressed as

$$
\begin{aligned}
d \log V(t) & =\kappa\left[\bar{\theta}+\frac{\varsigma_{\omega}}{\kappa} \omega(t)-\log V(t)\right] d t+\sqrt{\omega(t)} d W_{v}^{P}(t) \\
d \omega(t) & =-\bar{\lambda} \omega(t) d t+d \bar{Z}(t) .
\end{aligned}
$$

Since in this case the processes under the real and risk neutral measures are different, we will describe them separately.

\section{C.4.1 Risk neutral measure}

The conditional characteristic function simplifies to

$$
\phi_{L O U S V}(t, T, u)=\exp \left[\varphi_{L O U S V, 0}(\tau)+\varphi_{L O U S V, V}(\tau) \log V(t)+\varphi_{L O U S V, \omega}(\tau) \omega(t)\right]
$$

where

$$
\begin{aligned}
\varphi_{L O U S V, V}(\tau) & =i u \exp (-\kappa \tau), \\
\varphi_{L O U S V, \omega}(\tau) & =\frac{u^{2}}{2(2 \kappa-\lambda)}[\exp (-2 \kappa \tau)-\exp (-\lambda \tau)]
\end{aligned}
$$

and

$$
\varphi_{L O U S V, 0}(\tau)=i \bar{\theta} u[1-\exp (-\kappa \tau)]+\lambda[\varkappa(\tau, u)-\tau]
$$

with

$$
\varkappa(\tau, u)=\int_{0}^{\tau} \frac{\bar{\delta}}{\bar{\delta}-\frac{u^{2}}{2(2 \kappa-\lambda)}[\exp (-2 \kappa x)-\exp (-\lambda x)]} d x
$$

\section{C.4.2 Real measure}

We can write the characteristic function under the real measure as

$$
\begin{aligned}
& \phi_{\text {LOUSV }}^{P}(t, T, u)=E^{P}[\exp (i u \log V(T)+i v \omega(T)) \mid V(t), \omega(t)] \\
& =\exp \left[\varphi_{L O U S V, 0}^{P}(\tau)+\varphi_{L O U S V, V}^{P}(\tau) \log V(t)+\varphi_{L O U S V, \omega}^{P}(\tau) \omega(t)\right],
\end{aligned}
$$


where

$$
\begin{aligned}
& \varphi_{\text {LOUSV }, 0}^{P}(\tau)=i u \bar{\theta}[1-\exp (-\kappa \tau)]+\bar{\lambda}[\varkappa(\tau)-\tau], \\
& \varphi_{\text {LOUSV,V }}^{P}(\tau)=i u \exp (-\kappa \tau), \\
& \varphi_{\text {LOUSV }, \omega}^{P}(\tau)=i v \exp (-\bar{\lambda} \tau)-i u \frac{\varsigma_{\omega}}{\kappa-\bar{\lambda}}[\exp (-\kappa \tau)-\exp (-\bar{\lambda} \tau)] \\
& +\frac{u^{2}}{2(2 \kappa-\bar{\lambda})}[\exp (-2 \kappa \tau)-\exp (-\bar{\lambda} \tau)]
\end{aligned}
$$

and

$$
\varkappa(\tau)=\int_{0}^{\tau} \frac{\bar{\delta}}{\bar{\delta}-i v \exp (-\bar{\lambda} x)+i u \frac{\varsigma \omega[\exp (-\kappa x)-\exp (-\bar{\lambda} x)]}{\kappa-\bar{\lambda}}-\frac{u^{2}[\exp (-2 \kappa x)-\exp (-\bar{\lambda} x)]}{2(2 \kappa-\bar{\lambda})}} d x .
$$

Based on the characteristic function, it is possible to show that

$$
\begin{aligned}
& E[\log V(T) \mid V(t), \omega(t)]=\bar{\theta}[1-\exp (-\kappa \tau)] \\
& -\frac{\bar{\lambda}_{\omega}}{\bar{\delta}(\kappa-\bar{\lambda})}\left[\frac{1-\exp (-\kappa \tau)}{\kappa}-\frac{1-\exp (-\bar{\lambda} \tau)}{\bar{\lambda}}\right] \\
& +\exp (-\kappa \tau) \log V(t) \\
& -\frac{\varsigma_{\omega}}{\kappa-\bar{\lambda}}[\exp (-\kappa \tau)-\exp (-\bar{\lambda} \tau)] \omega(t) \\
& E[\omega(T) \mid V(t), \omega(t)]=\frac{1}{\bar{\delta}}[1-\exp (-\bar{\lambda} \tau)]+\exp (-\bar{\lambda} \tau) \omega(t), \\
& V[\log V(T) \mid V(t), \omega(t)]=\frac{\bar{\lambda}}{(2 \kappa-\bar{\lambda}) \bar{\delta}}\left[\frac{1-\exp (-\bar{\lambda} \tau)}{\bar{\lambda}}-\frac{1-\exp (-2 \kappa \tau)}{2 \kappa}\right] \\
& +\frac{2 \varsigma_{\omega}^{2} \bar{\lambda}}{\bar{\delta}^{2}(\kappa-\bar{\lambda})^{2}}\left[\frac{1-\exp (-2 \kappa \tau)}{2 \kappa}+\frac{1-\exp (-2 \bar{\lambda} \tau)}{2 \bar{\lambda}}-2 \frac{1-\exp (-(\kappa+\bar{\lambda}) \tau)}{\kappa+\bar{\lambda}}\right] \\
& +\frac{1}{2 \kappa-\bar{\lambda}}[\exp (-\bar{\lambda} \tau)-\exp (-2 \kappa \tau)] \omega(t) \\
& V^{P}[\omega(T) \mid V(t), \omega(t)]=\frac{1-\exp (-2 \bar{\lambda} \tau)}{\bar{\delta}^{2}},
\end{aligned}
$$

and

$$
\operatorname{cov}[\log V(T), \omega(T) \mid V(t), \omega(t)]=-\frac{2 \varsigma_{\omega} \bar{\lambda}}{\bar{\delta}^{2}(\kappa-\bar{\lambda})}\left[\frac{1-\exp (-(\kappa+\bar{\lambda}) \tau)}{\kappa+\bar{\lambda}}-\frac{1-\exp (-2 \bar{\lambda} \tau)}{2 \bar{\lambda}}\right]
$$

\section{C.5 Central tendency and jumps}

For this case we use the same prices of risk as in the CTOU model and assume that jump risk is not priced. The, we obtain

$$
\begin{aligned}
d \log V(t) & =\kappa\left[\theta^{P}(t)-\log V(t)\right] d t+\sigma d W_{v}^{P}(t)+d Z(t)-\frac{\lambda}{\kappa \delta} d t, \\
d \theta^{P}(t) & =\bar{\kappa}\left[\bar{\theta}^{P}-\theta^{P}(t)\right] d t+\bar{\sigma} d W_{\theta}^{P}(t),
\end{aligned}
$$


under the real measure, where

$$
\bar{\theta}^{P}=\bar{\theta}+\frac{\sigma \varsigma}{\kappa}+\frac{\bar{\sigma} \bar{\varsigma}}{\bar{\kappa}},
$$

and

$$
\theta^{P}(t)=\theta(t)+\frac{\sigma \varsigma}{\kappa} .
$$

The conditional characteristic function becomes

$$
\begin{aligned}
\phi_{\text {CTOUJ }}(t, T, u) & =E[\exp (i u \log V(T)+i v \theta(T) \mid V(t), \theta(t)] \\
& =\exp \left[\varphi_{\text {CTOU J }, 0}(\tau)+\varphi_{\text {CTOU }, \theta}(\tau) \theta(t)+\varphi_{\text {CTOU J }, V}(\tau) \log V(t)\right],
\end{aligned}
$$

where

$$
\begin{gathered}
\varphi_{\text {CTOU }, 0}(\tau)=i v \bar{\theta}[1-\exp (-\bar{\kappa} \tau)] \\
+i u\left(\bar{\theta}-\frac{\lambda}{\kappa \delta}\right) \frac{\kappa \bar{\kappa}}{\kappa-\bar{\kappa}}\left[\frac{1-\exp (-\bar{\kappa} \tau)}{\bar{\kappa}}-\frac{1-\exp (-\kappa \tau)}{\kappa}\right] \\
-\frac{\bar{\sigma}^{2} v^{2}}{4 \bar{\kappa}}[1-\exp (-2 \bar{\kappa} \tau)]-\frac{\sigma^{2} u^{2}}{4 \kappa}[1-\exp (-2 \kappa \tau)] \\
-\frac{\bar{\sigma}^{2} u^{2}}{2}\left(\frac{\kappa}{\kappa-\bar{\kappa}}\right)^{2}\left[\frac{1-\exp (-2 \bar{\kappa} \tau)}{2 \bar{\kappa}}+\frac{1-\exp (-2 \kappa \tau)}{2 \kappa}-2 \frac{1-\exp (-(\kappa+\bar{\kappa}) \tau)}{\kappa+\bar{\kappa}}\right] \\
-u v \bar{\sigma}^{2} \frac{\kappa}{\kappa-\bar{\kappa}}\left[\frac{1-\exp (-2 \bar{\kappa} \tau)}{2 \bar{\kappa}}-\frac{1-\exp (-(\kappa+\bar{\kappa}) \tau)}{\kappa+\bar{\kappa}}\right] \\
+\frac{\lambda}{\kappa} \log \left[\frac{\delta-i u \exp (-\kappa \tau)}{\delta-i u}\right] \\
-i u \frac{\lambda}{\kappa \delta} \frac{\kappa}{\kappa-\bar{\kappa}}[\exp (-\bar{\kappa} \tau)-\exp (-\kappa \tau)], \\
\varphi_{\text {CTOU }, \theta}(\tau)=i v \exp (-\bar{\kappa} \tau)+i u \frac{\kappa}{\kappa-\bar{\kappa}}[\exp (-\bar{\kappa} \tau)-\exp (-\kappa \tau)]
\end{gathered}
$$

and

$$
\varphi_{C T O U J, V}(\tau)=i u \exp (-\kappa \tau) .
$$

Using the characteristic function, we can show that

$$
\begin{gathered}
E[\log V(T) \mid V(t), \theta(t)]=\bar{\theta}\left[1-\exp (-\kappa \tau)-\frac{\kappa}{\kappa-\bar{\kappa}}(\exp (-\bar{\kappa} \tau)-\exp (-\kappa \tau))\right] \\
+\frac{\kappa}{\kappa-\bar{\kappa}}[\exp (-\bar{\kappa} \tau)-\exp (-\kappa \tau)] \theta(t)+\exp (-\kappa \tau) \log V(t), \\
V[\log V(T) \mid V(t), \theta(t)]=\left(\frac{\sigma^{2}}{2 \kappa}+\frac{\lambda}{\kappa \delta^{2}}\right)[1-\exp (-2 \kappa \tau)] \\
+\bar{\sigma}^{2}\left(\frac{\kappa}{\kappa-\bar{\kappa}}\right)^{2}\left[\frac{1-\exp (-2 \bar{\kappa} \tau)}{2 \bar{\kappa}}+\frac{1-\exp (-2 \kappa \tau)}{2 \kappa}-2 \frac{1-\exp (-(\kappa+\bar{\kappa}) \tau)}{\kappa+\bar{\kappa}}\right], \\
V[\log V(T), \theta(T) \mid V(t), \theta(t)]=\bar{\sigma}^{2} \frac{\kappa}{\kappa-\bar{\kappa}}\left[\frac{1-\exp (-2 \bar{\kappa} \tau)}{2 \bar{\kappa}}-\frac{1-\exp (-(\kappa+\bar{\kappa}) \tau)}{\kappa+\bar{\kappa}}\right] .
\end{gathered}
$$




\section{C.6 Central tendency and stochastic volatility}

The price of risk is such that $d W_{v}^{Q}(t)=d W_{v}^{P}(t)+\varsigma_{\omega} \sqrt{\omega(t)} d t$ and $d W_{\theta}^{Q}(t)=d W_{\theta}^{P}(t)+$ $\bar{\varsigma} d t$, which yields

$$
\begin{aligned}
d \log V(t) & =\kappa\left[\theta(t)+\frac{\varsigma_{\omega}}{\kappa} \omega(t)-\log V(t)\right] d t+\sqrt{\omega(t)} d W_{v}^{P}(t), \\
d \theta(t) & =\bar{\kappa}\left[\bar{\theta}^{P}-\theta(t)\right] d t+\bar{\sigma} d W_{\theta}^{P}(t), \\
d \omega(t) & =-\bar{\lambda} \omega(t) d t+d \bar{Z}(t),
\end{aligned}
$$

under the real measure, where

$$
\bar{\theta}^{P}=\bar{\theta}+\frac{\bar{\varsigma} \bar{\sigma}}{\bar{\kappa}} .
$$

\section{C.6.1 Risk neutral measure}

The conditional characteristic function is

$$
\phi_{C T O U S V}(t, T, u)=\exp \left[\begin{array}{c}
\varphi_{C T O U S V, 0}(\tau)+\varphi_{C T O U S V, V}(\tau) \log V(t) \\
+\varphi_{C T O U S V, \omega}(\tau) \omega(t)+\varphi_{C T O U S V, \theta}(\tau) \theta(t)
\end{array}\right]
$$

where

$$
\begin{aligned}
\varphi_{\text {CTOUSV }, V}(\tau) & =i u \exp (-\kappa \tau), \\
\varphi_{\text {CTOUSV }, \omega}(\tau) & =\frac{u^{2}}{2(2 \kappa-\lambda)}[\exp (-2 \kappa \tau)-\exp (-\lambda \tau)], \\
\varphi_{C T O U S V, \theta}(\tau) & =i u \frac{\kappa}{\kappa-\bar{\kappa}}[\exp (-\bar{\kappa} \tau)-\exp (-\kappa \tau)],
\end{aligned}
$$

and

$$
\begin{aligned}
\varphi_{\text {CTOUSV }, 0}(\tau)= & i u \bar{\kappa} \bar{\theta} \frac{\kappa}{\kappa-\bar{\kappa}}\left[\frac{1-\exp (-\bar{\kappa} \tau)}{\bar{\kappa}}-\frac{1-\exp (-\kappa \tau)}{\kappa}\right] \\
& -\frac{1}{2} \bar{\sigma}^{2} u^{2}\left(\frac{\kappa}{\kappa-\bar{\kappa}}\right)^{2}\left[\begin{array}{c}
\frac{1-\exp (-2 \bar{\kappa} \tau)}{2 \bar{\kappa}}+\frac{1-\exp (-2 \kappa \tau)}{2 \kappa} \\
-2 \frac{1-\exp (-(\kappa+\bar{\kappa}) \tau)}{\kappa+\bar{\kappa}}
\end{array}\right] \\
& +\lambda[\varkappa(\tau, u)-\tau],
\end{aligned}
$$

with $\varkappa(\tau, u)$ defined in $(\mathrm{C} 3)$.

\section{C.6.2 Real measure}

We can write the characteristic function under the real measure as

$$
\begin{gathered}
\phi^{P}\left(t, T, u_{1}, u_{2}, u_{3}\right)=E\left[\exp \left[i u_{1} \log V(T)+i u_{2} \theta(T)+i u_{3} \omega(T)\right] \mid V(t), \theta(t), \omega(t)\right] \\
=\exp \left[\varphi_{\text {CTOUSV }, 0}^{P}(\tau)+\varphi_{C T O U S V, V}^{P}(\tau) \log V(t)+\varphi_{C T O U S V, \theta}^{P}(\tau) \theta(t)+\varphi_{C T O U S V, \omega}^{P}(\tau) \omega(t)\right],
\end{gathered}
$$


where

$$
\begin{gathered}
\varphi_{\text {CTOUSV }, 0}^{P}(\tau)=i u_{2} \bar{\theta}(1-\exp (-\bar{\kappa} \tau)) \\
+i u_{1} \bar{\theta} \frac{\kappa \bar{\kappa}}{\kappa-\bar{\kappa}}\left[\frac{1-\exp (-\bar{\kappa} \tau)}{\bar{\kappa}}-\frac{1-\exp (-\kappa \tau)}{\kappa}\right] \\
-u_{2}^{2} \frac{\bar{\sigma}^{2}}{2} \frac{1-\exp (-2 \bar{\kappa} \tau)}{2 \bar{\kappa}} \\
-u_{1}^{2} \frac{\bar{\sigma}^{2}}{2}\left(\frac{\kappa}{\kappa-\bar{\kappa}}\right)^{2}\left[\frac{1-\exp (-2 \bar{\kappa} \tau)}{2 \bar{\kappa}}+\frac{1-\exp (-2 \kappa \tau)}{2 \kappa}-2 \frac{1-\exp (-(\kappa+\bar{\kappa}) \tau)}{\kappa+\bar{\kappa}}\right] \\
-u_{1} u_{2} \bar{\sigma}^{2} \frac{\kappa}{\kappa-\bar{\kappa}}\left[\frac{1-\exp (-2 \bar{\kappa} \tau)}{2 \bar{\kappa}}-\frac{1-\exp (-(\kappa+\bar{\kappa}) \tau)}{\kappa+\bar{\kappa}}\right] \\
+\bar{\lambda}[\varkappa(\tau)-\tau], \\
\varphi_{C T O U S V, V}^{P}(\tau)=i u_{1} \exp (-\kappa \tau), \\
\varphi_{C T O U S V, \omega}^{P}(\tau)=i u_{3}^{P}(\tau)=i u_{2} \exp (-\bar{\kappa} \tau)+i u_{1} \frac{\kappa}{\kappa-\bar{\kappa}}[\exp (-\bar{\kappa} \tau)-\exp (-\kappa \tau)] \\
+\frac{u_{1}^{2}}{2(2 \kappa-\bar{\lambda})}[\exp (-2 \kappa \tau)-\exp (-\bar{\lambda} \tau)]
\end{gathered}
$$

and

$$
\varkappa(\tau)=\int_{0}^{\tau} \frac{\delta}{\delta-i u_{3} \exp (-\bar{\lambda} x)+i u_{1} \frac{{ }_{\omega}[\exp (-\kappa x)-\exp (-\bar{\lambda} x)]}{\kappa-\lambda}-\frac{u_{1}^{2}[\exp (-2 \kappa x)-\exp (-\bar{\lambda} x)]}{2(2 \kappa-\bar{\lambda})}} d x .
$$

Based on the characteristic function, it is possible to show that

$$
\begin{aligned}
E[\log V(T) \mid V(t), \theta(t), \omega(t)]= & \bar{\theta}^{P} \frac{\kappa \bar{\kappa}}{\kappa-\bar{\kappa}}\left[\frac{1-\exp (-\bar{\kappa} \tau)}{\bar{\kappa}}-\frac{1-\exp (-\kappa \tau)}{\kappa}\right] \\
& -\frac{\bar{\lambda} \varsigma_{\omega}}{\bar{\delta}(\kappa-\bar{\lambda})}\left[\frac{1-\exp (-\kappa \tau)}{\kappa}-\frac{1-\exp (-\bar{\lambda} \tau)}{\bar{\lambda}}\right] \\
& +\exp (-\kappa \tau) \log V(t) \\
& +\frac{\kappa}{\kappa-\bar{\kappa}}[\exp (-\bar{\kappa} \tau)-\exp (-\kappa \tau)] \theta(t) \\
& -\frac{\varsigma_{\omega}}{\kappa-\bar{\lambda}}[\exp (-\kappa \tau)-\exp (-\bar{\lambda} \tau)] \omega(t) \\
E[\theta(T) \mid V(t), \theta(t), \omega(t)]= & \bar{\theta}^{P}[1-\exp (-\bar{\kappa} \tau)]+\exp (-\bar{\kappa} \tau) \theta(t) \\
E[\omega(T) \mid V(t), \theta(t), \omega(t)]= & \frac{1}{\bar{\delta}}[1-\exp (-\bar{\lambda} \tau)]+\exp (-\bar{\lambda} \tau) \omega(t),
\end{aligned}
$$




$$
\begin{gathered}
V[\log V(T) \mid V(t), \theta(t), \omega(t)]=\frac{\bar{\lambda}}{(2 \kappa-\bar{\lambda}) \bar{\delta}}\left[\frac{1-\exp (-\bar{\lambda} \tau)}{\bar{\lambda}}-\frac{1-\exp (-2 \kappa \tau)}{2 \kappa}\right] \\
+\bar{\sigma}^{2}\left(\frac{\kappa}{\kappa-\bar{\kappa}}\right)^{2}\left[\frac{1-\exp (-2 \bar{\kappa} \tau)}{2 \bar{\kappa}}+\frac{1-\exp (-2 \kappa \tau)}{2 \kappa}-2 \frac{1-\exp (-(\kappa+\bar{\kappa}) \tau)}{\kappa+\bar{\kappa}}\right] \\
+\frac{2 \varsigma_{\omega}^{2} \bar{\lambda}}{\bar{\delta}^{2}(\kappa-\bar{\lambda})^{2}}\left[\frac{1-\exp (-2 \kappa \tau)}{2 \kappa}+\frac{1-\exp (-2 \bar{\lambda} \tau)}{2 \bar{\lambda}}-2 \frac{1-\exp (-(\kappa+\bar{\lambda}) \tau)}{\kappa+\bar{\lambda}}\right] \\
+\frac{1}{2 \kappa-\bar{\lambda}}[\exp (-\bar{\lambda} \tau)-\exp (-2 \kappa \tau)] \omega(t), \\
V[\theta(T) \mid V(t), \theta(t), \omega(t)]=\bar{\sigma}^{2} \frac{1-\exp (-2 \bar{\kappa} \tau)}{2 \bar{\kappa}} \\
V[\omega(T) \mid V(t), \theta(t), \omega(t)]=\frac{1-\exp (-2 \bar{\lambda} \tau)}{\bar{\delta}^{2}}, \\
\operatorname{cov}[\log V(T), \theta(T) \mid V(t), \theta(t), \omega(t)]=\frac{\bar{\sigma}^{2} \kappa}{\kappa-\bar{\kappa}}\left[\frac{1-\exp (-2 \bar{\kappa} \tau)}{2 \bar{\kappa}}-\frac{1-\exp (-(\kappa+\bar{\kappa}) \tau)}{\kappa+\bar{\kappa}}\right]
\end{gathered}
$$

and

$\operatorname{cov}[\log V(T), \omega(T) \mid V(t), \theta(t), \omega(t)]=-\frac{2 \varsigma_{\omega} \bar{\lambda}}{\bar{\delta}^{2}(\kappa-\bar{\lambda})}\left[\frac{1-\exp (-(\kappa+\bar{\lambda}) \tau)}{\kappa+\bar{\lambda}}-\frac{1-\exp (-2 \bar{\lambda} \tau)}{2 \bar{\lambda}}\right]$

\section{One-factor models variances and autocorrelations}

Let $V(t)$ follow the SQR process. Then, it can be shown that

$$
V[V(T) \mid V(t)]=\frac{\sigma^{2}}{2 \kappa}(1-\exp (-\kappa \tau))^{2}+\frac{\sigma^{2}}{\kappa} \exp (-\kappa \tau)(1-\exp (-\kappa \tau)) V(t),
$$

and $\operatorname{corr}[V(T), V(t)]=\exp (-\kappa \tau)$.

Alternatively, if $V(t)$ follows the LOU process it holds that

$$
\begin{aligned}
V[V(T) \mid V(t)]= & \exp \left[2 \bar{\theta}(1-\exp (-\kappa \tau))+\frac{\sigma^{2}}{2 \kappa}(1-\exp (-2 \kappa \tau))\right] \\
& \times\left[\exp \left[\frac{\sigma^{2}}{2 \kappa}(1-\exp (-2 \kappa \tau))\right]-1\right][V(t)]^{2 \exp (-\kappa \tau)},
\end{aligned}
$$

and

$$
\operatorname{corr}[V(T), V(t)]=\frac{\exp \left[\frac{\sigma^{2}}{2 \kappa} \exp (-\kappa \tau)\right]-1}{\exp \left[\frac{\sigma^{2}}{2 \kappa}\right]-1}
$$


Table 1

Root mean square pricing errors in existing one-factor models

\begin{tabular}{|c|c|c|c|}
\hline & \multicolumn{2}{|c|}{ Aug08-Mar10 estimates } & Full sample estimates \\
\hline & In-sample (Feb06-Aug08) & Out-of-sample (Aug08-Dec10) & Feb06-Dec10 \\
\hline SQR & 0.807 & 2.037 & 2.454 \\
\hline LOU & 0.779 & 1.982 & 1.533 \\
\hline
\end{tabular}

Notes: "SQR" denotes square root model while "LOU" refers to the log-normal Ornstein-Uhlenbeck process. 
Table 2

Parameters estimates of the existing one-factor models

\begin{tabular}{|c|c|c|c|c|c|c|c|c|c|c|}
\hline \multirow[b]{2}{*}{ Model } & \multirow[b]{2}{*}{ Estimation } & \multicolumn{3}{|c|}{ Real measure parameters } & \multicolumn{6}{|c|}{ Pricing error parameters } \\
\hline & & $\kappa$ & $\bar{\theta}$ & $\sigma$ & $\sigma_{f \varepsilon}$ & $\sigma_{f \xi}$ & $\sigma_{a}$ & $\eta_{o}$ & $\sigma_{b}$ & $\sigma_{c}$ \\
\hline \multirow[t]{2}{*}{ SQR } & Full Sample & $\begin{array}{c}2.555 \\
(0.071)\end{array}$ & $\begin{array}{l}24.350 \\
(0.661)\end{array}$ & $\begin{array}{c}4.504 \\
(0.005)\end{array}$ & $\begin{array}{c}0.122 \\
(0.001)\end{array}$ & $\begin{array}{c}0.081 \\
(0.009)\end{array}$ & $\begin{array}{c}0.006 \\
\left(2810^{-4}\right)\end{array}$ & $\begin{array}{c}0.497 \\
(0.019)\end{array}$ & $\begin{array}{c}0.437 \\
(0.017)\end{array}$ & $\begin{array}{l}-0.901 \\
(0.002)\end{array}$ \\
\hline & Until Aug 08 & $\begin{array}{c}1.495 \\
(0.015)\end{array}$ & $\begin{array}{l}19.666 \\
(0.150)\end{array}$ & $\begin{array}{c}2.916 \\
(0.006)\end{array}$ & $\begin{array}{c}0.040 \\
\left(4.110^{-4}\right)\end{array}$ & $\begin{array}{c}0.008 \\
(0.001)\end{array}$ & $\begin{array}{c}0.006 \\
\left(2.610^{-4}\right)\end{array}$ & $\begin{array}{c}0.369 \\
(0.024)\end{array}$ & $\begin{array}{c}0.275 \\
(0.009)\end{array}$ & $\begin{array}{l}-0.727 \\
(0.003)\end{array}$ \\
\hline \multirow[t]{2}{*}{ LOU } & Full Sample & $\begin{array}{c}2.898 \\
(0.004)\end{array}$ & $\begin{array}{c}3.099 \\
(0.112)\end{array}$ & $\begin{array}{c}0.884 \\
\left(4.410^{-4}\right)\end{array}$ & $\begin{array}{c}0.075 \\
(0.001)\end{array}$ & $\begin{array}{c}0.030 \\
(0.003)\end{array}$ & $\begin{array}{c}0.006 \\
\left(2.410^{-4}\right)\end{array}$ & $\begin{array}{c}0.455 \\
(0.017)\end{array}$ & $\begin{array}{c}0.207 \\
(0.007)\end{array}$ & $\begin{array}{l}-0.901 \\
(0.002)\end{array}$ \\
\hline & Until Aug 08 & $\begin{array}{c}1.732 \\
(0.009)\end{array}$ & $\begin{array}{c}2.927 \\
(0.171)\end{array}$ & $\begin{array}{c}0.710 \\
(0.001)\end{array}$ & $\begin{array}{c}0.042 \\
\left(4.210^{-4}\right)\end{array}$ & $\begin{array}{c}0.008 \\
(0.001)\end{array}$ & $\begin{array}{c}0.005 \\
\left(2.210^{-4}\right)\end{array}$ & $\begin{array}{c}0.412 \\
(0.021)\end{array}$ & $\begin{array}{c}0.174 \\
(0.006)\end{array}$ & $\begin{array}{l}-0.773 \\
(0.005)\end{array}$ \\
\hline
\end{tabular}

Notes: "SQR" denotes square root model while "LOU" refers to the log-normal Ornstein-Uhlenbeck process. Standard errors, displayed in parentheses, have been obtained by using the outer-product of the score to estimate the information matrix. $\sigma_{f \varepsilon}$ and $\sigma_{f \xi}$ are the parameters of the futures pricing errors. $\eta_{o}, \sigma_{a}, \sigma_{b}$ and $\sigma_{c}$ are the parameters of the option pricing errors, whose variance is the quadratic function of moneyness (3). 
Table 3

Root mean square pricing errors in the extended models

Aug08-Mar10 estimates

Full sample estimates

\begin{tabular}{lccc} 
& In-sample (Feb06-Aug08) & Out-of-sample (Aug08-Dec10) & Feb06-Dec10 \\
\hline CTOU & 0.348 & 0.713 & 0.655 \\
LOUJ & 0.837 & 2.160 & 1.634 \\
LOUSV & 0.664 & 1.617 & 1.303 \\
CTOUJ & 0.354 & 0.691 & 0.631 \\
CTOUSV & 0.232 & 0.344 & 0.306 \\
CSQR & 0.424 & 1.156 & 0.691 \\
\hline
\end{tabular}

Notes: "SQR" denotes square root model and "LOU" refers to a log-normal Ornstein-Uhlenbeck process. "LOUJ" introduces jumps in the LOU model, whose size follows an exponential distribution. "CTOU" adds central tendency to the LOU process. "LOUSV" denotes a LOU model with stochastic volatility modelled with a Gamma OU Lévy process. "CTOUJ" adds central tendency and jumps to the LOU model, while "CTOUSV" introduces central tendency and stochastic volatility. "CSQR" is the concatenated SQR model. 
Table 4. Real measure parameters estimates of the extended models

\begin{tabular}{|c|c|c|c|c|c|c|c|c|c|c|}
\hline Extension & Estimation & $\kappa$ & $\bar{\kappa}$ & $\bar{\theta}$ & $\lambda$ & $\delta$ & $\sigma$ & $\bar{\sigma}$ & $\bar{\lambda}$ & $\bar{\delta}$ \\
\hline \multirow{4}{*}{ CTOU } & Full Sample & 5.827 & 0.300 & 3.019 & & & 1.037 & 0.446 & & \\
\hline & & $(0.017)$ & $(0.003)$ & $(0.710)$ & & & $(0.001)$ & $(0.001)$ & & \\
\hline & Until Aug08 & 6.946 & 0.422 & 2.851 & & & 1.012 & 0.447 & & \\
\hline & & $(0.041)$ & $(0.006)$ & $(0.867)$ & & & $(0.002)$ & $(0.001)$ & & \\
\hline \multirow[t]{4}{*}{ LOUJ } & Full Sample & 3.632 & & 3.093 & 5.370 & 4.870 & 0.728 & & & \\
\hline & & $(0.004)$ & & $(0.112)$ & $(0.109)$ & $(0.037)$ & $(0.002)$ & & & \\
\hline & Until Aug08 & 2.289 & & 2.897 & 14.621 & 7.802 & 0.382 & & & \\
\hline & & $(0.008)$ & & $(0.159)$ & $(0.721)$ & $(0.149)$ & $(0.010)$ & & & \\
\hline \multirow[t]{4}{*}{ LOUSV } & Full Sample & 0.679 & & 2.978 & & & & & 14.937 & 3.026 \\
\hline & & $(0.004)$ & & $(0.002)$ & & & & & $(0.038)$ & $(0.007)$ \\
\hline & Until Aug08 & 0.573 & & 2.842 & & & & & 15.631 & 3.474 \\
\hline & & $(0.005)$ & & $(0.002)$ & & & & & $(0.126)$ & $(0.014)$ \\
\hline \multirow[t]{4}{*}{ CTOUJ } & Full Sample & 5.452 & 0.285 & 3.021 & 6.671 & 5.196 & 0.836 & 0.486 & & \\
\hline & & $(0.011)$ & $(0.003)$ & $(0.873)$ & $(0.071)$ & $(0.013)$ & $(0.002)$ & $(0.001)$ & & \\
\hline & Until Aug08 & 6.094 & 0.360 & 2.861 & 11.382 & 5.962 & 0.640 & 0.450 & & \\
\hline & & $(0.029)$ & $(0.006)$ & $(1.055)$ & $(0.222)$ & $(0.030)$ & $(0.004)$ & $(0.001)$ & & \\
\hline \multirow[t]{4}{*}{ CTOUSV } & Full Sample & 8.903 & 0.357 & 2.934 & & & & 0.222 & 2.140 & 0.271 \\
\hline & & (0.009) & $(0.002)$ & $(0.163)$ & & & & $(0.001)$ & $(0.005)$ & $(0.001)$ \\
\hline & Until Aug08 & 10.029 & 0.477 & 2.826 & & & & 0.227 & 0.953 & 0.170 \\
\hline & & $(0.038)$ & $(0.006)$ & $(0.209)$ & & & & $(0.003)$ & $(0.013)$ & $(0.001)$ \\
\hline \multirow[t]{4}{*}{ CSQR } & Full Sample & 3.076 & 0.673 & 22.476 & & & 6.424 & 2.145 & & \\
\hline & & $(0.009)$ & $(0.021)$ & $(0.645)$ & & & $(0.005)$ & $(0.007)$ & & \\
\hline & Until Aug08 & 2.575 & 0.446 & 19.795 & & & 3.732 & 1.360 & & \\
\hline & & $(0.126)$ & $(0.032)$ & $(1.565)$ & & & $(0.006)$ & $(0.013)$ & & \\
\hline
\end{tabular}

Notes: "SQR" denotes square root model and "LOU" refers to a log-normal Ornstein-Uhlenbeck process. "LOUJ" introduces jumps in the LOU model, whose size follows an exponential distribution. "CTOU" adds central tendency to the LOU process. "LOUSV" denotes a LOU model with stochastic volatility modelled with a Gamma OU Lévy process. "CTOUJ" adds central tendency and jumps to the LOU model, while "CTOUSV" introduces central tendency and stochastic volatility. "CSQR" is the concatenated SQR model. Standard errors, displayed in parentheses, have been obtained by using the outer-product of the score to estimate the information matrix. 
Table 5

Prices of risk in the CTOUSV model

\begin{tabular}{cccccccc} 
& \multicolumn{2}{c}{ Estimates } & & \multicolumn{3}{c}{ Wald tests } \\
\cline { 2 - 3 } \cline { 5 - 7 } & $\varsigma_{\omega}$ & $\bar{\varsigma}$ & & $\varsigma_{\omega}=0$ & $\bar{\varsigma}=0$ & Joint \\
\hline Full sample & -1.27 & 0.15 & & 76.35 & 0.32 & 76.99 \\
& $(0.15)$ & $(0.26)$ & & $(0.00)$ & $(0.57)$ & $(0.00)$ \\
Until Aug 08 & -1.19 & 0.14 & & 45.63 & 0.11 & 46.67 \\
& $(0.18)$ & $(0.44)$ & & $(0.00)$ & $(0.74)$ & $(0.00)$ \\
\hline
\end{tabular}

Notes: standard errors are displayed in parentheses below the estimates, and p-values are reported below the Wald tests. "CTOUSV" introduces central tendency and stochastic volatility in a lognormal Ornstein-Uhlenbeck process. 
Table 6

Root mean square pricing errors of futures prices by maturity

\begin{tabular}{lccccccccc} 
Maturity & $\mathrm{N}$ & SQR & LOU & CTOU & LOUJ & LOUSV & CTOUJ & CTOUSV & CSQR \\
\hline Less than 1 month & 1036 & 2.607 & 1.957 & 1.606 & 2.068 & 2.552 & 1.652 & 0.982 & 1.109 \\
From 1 to 3 months & 2290 & 6.128 & 3.953 & 2.046 & 4.334 & 4.332 & 2.115 & 0.844 & 1.029 \\
From 3 to 6 months & 3093 & 9.039 & 5.250 & 1.561 & 5.755 & 4.797 & 1.626 & 0.494 & 0.696 \\
More than 6 months & 2246 & 9.370 & 6.054 & 1.278 & 6.489 & 4.031 & 1.341 & 0.567 & 0.743 \\
Total & 8665 & 7.916 & 4.892 & 1.645 & 5.311 & 4.262 & 1.708 & 0.688 & 0.862 \\
\hline
\end{tabular}

Notes: "SQR" denotes square root model and "LOU" refers to a log-normal Ornstein-Uhlenbeck process. "LOUJ" introduces jumps in the LOU model, whose size follows an exponential distribution. "CTOU" adds central tendency to the LOU process. "LOUSV" denotes a LOU model with stochastic volatility modelled with a Gamma OU Lévy process "CTOUJ" adds central tendency and jumps to the LOU model, while "CTOUSV" introduces central tendency and stochastic volatility. "CSQR" is the concatenated SQR model. The column labeled N gives the number of prices per category. Results are based on full sample estimates. 
Table 7

Root mean square pricing errors of call prices by moneyness and maturities

\begin{tabular}{cccccccccc} 
& \multicolumn{10}{c}{$(\mathrm{a}) \tau<1$ month } \\
Moneyness & $\mathrm{N}$ & SQR & LOU & CTOU & LOUJ & LOUSV & CTOUJ & CTOUSV & CSQR \\
\hline$<-0.3$ & 2199 & 0.155 & 0.138 & 0.133 & 0.142 & 0.110 & 0.204 & 0.142 & 0.214 \\
{$[-0.3,-0.1)$} & 2080 & 0.516 & 0.373 & 0.352 & 0.381 & 0.140 & 0.375 & 0.237 & 0.601 \\
{$[-0.1,0.1)$} & 2260 & 0.867 & 0.597 & 0.527 & 0.531 & 0.245 & 0.509 & 0.227 & 0.800 \\
{$[0.1,0.3)$} & 2254 & 0.771 & 0.615 & 0.535 & 0.414 & 0.344 & 0.409 & 0.187 & 0.640 \\
$\geq 0.3$ & 3085 & 0.389 & 0.361 & 0.338 & 0.214 & 0.253 & 0.213 & 0.160 & 0.349 \\
\hline
\end{tabular}

(b) 1 month $<\tau<3$ months

\begin{tabular}{cccccccccc} 
Moneyness & $\mathrm{N}$ & SQR & LOU & CTOU & LOUJ & LOUSV & CTOUJ & CTOUSV & CSQR \\
\hline$<-0.3$ & 3553 & 0.250 & 0.176 & 0.177 & 0.174 & 0.151 & 0.253 & 0.214 & 0.390 \\
{$[-0.3,-0.1)$} & 4217 & 0.560 & 0.381 & 0.384 & 0.394 & 0.166 & 0.372 & 0.270 & 0.768 \\
{$[-0.1,0.1)$} & 4701 & 0.787 & 0.494 & 0.486 & 0.495 & 0.199 & 0.476 & 0.173 & 0.792 \\
{$[0.1,0.3)$} & 4638 & 0.864 & 0.573 & 0.557 & 0.452 & 0.368 & 0.434 & 0.192 & 0.704 \\
$\geq 0.3$ & 8089 & 0.573 & 0.448 & 0.443 & 0.272 & 0.328 & 0.261 & 0.178 & 0.482 \\
\hline
\end{tabular}

\begin{tabular}{|c|c|c|c|c|c|c|c|c|c|}
\hline \multicolumn{10}{|c|}{ (c) $\tau>3$ months } \\
\hline Moneyness & $\mathrm{N}$ & SQR & $\mathrm{LOU}$ & CTOU & LOUJ & LOUSV & CTOUJ & CTOUSV & CSQR \\
\hline$<-0.3$ & 3229 & 0.321 & 0.198 & 0.215 & 0.187 & 0.195 & 0.251 & 0.250 & 0.606 \\
\hline$[-0.3,-0.1)$ & 3719 & 0.486 & 0.360 & 0.356 & 0.370 & 0.222 & 0.351 & 0.280 & 0.806 \\
\hline$[-0.1,0.1)$ & 4491 & 0.614 & 0.451 & 0.426 & 0.430 & 0.254 & 0.407 & 0.256 & 0.741 \\
\hline$[0.1,0.3)$ & 3971 & 0.860 & 0.608 & 0.583 & 0.455 & 0.401 & 0.432 & 0.297 & 0.734 \\
\hline$\geq 0.3$ & 5613 & 0.758 & 0.564 & 0.555 & 0.312 & 0.395 & 0.319 & 0.289 & 0.648 \\
\hline \multicolumn{10}{|c|}{ (d) All maturities } \\
\hline Moneyness & $\mathrm{N}$ & SQR & $\mathrm{LOU}$ & CTOU & LOUJ & LOUSV & CTOUJ & CTOUSV & CSQR \\
\hline$<-0.3$ & 8981 & 0.260 & 0.176 & 0.183 & 0.171 & 0.160 & 0.241 & 0.213 & 0.451 \\
\hline$[-0.3,-0.1)$ & 10016 & 0.524 & 0.372 & 0.367 & 0.382 & 0.185 & 0.365 & 0.267 & 0.751 \\
\hline$[-0.1,0.1)$ & 11452 & 0.742 & 0.500 & 0.472 & 0.479 & 0.231 & 0.457 & 0.219 & 0.774 \\
\hline$[0.1,0.3)$ & 10863 & 0.844 & 0.595 & 0.562 & 0.446 & 0.376 & 0.428 & 0.235 & 0.702 \\
\hline$\geq 0.3$ & 16787 & 0.615 & 0.476 & 0.467 & 0.277 & 0.340 & 0.274 & 0.219 & 0.524 \\
\hline
\end{tabular}

Notes: Moneyness is defined as $\log (K / F(t, T))$, where $K$ and $F(t, T)$ are the strike and futures prices, respectively. "SQR" denotes square root model and "LOU" refers to a log-normal Ornstein-Uhlenbeck process. "LOUJ" introduces jumps in the LOU model, whose size follows an exponential distribution. "CTOU" adds central tendency to the LOU process. "LOUSV" denotes a LOU model with stochastic volatility modelled with a Gamma OU Lévy process. "CTOUJ" adds central tendency and jumps to the LOU model, while "CTOUSV" introduces central tendency and stochastic volatility. "CSQR" is a concatenated SQR model. The column labeled $\mathrm{N}$ gives the number of prices per category. $\tau$ denotes time to maturity. Results are based on full sample estimates. 
Table 8

Root mean square pricing errors of put prices by moneyness and maturities

\begin{tabular}{cccccccccc} 
& \multicolumn{10}{c}{$(\mathrm{a}) \tau<1$ month } \\
Moneyness & $\mathrm{N}$ & SQR & LOU & CTOU & LOUJ & LOUSV & CTOUJ & CTOUSV & CSQR \\
\hline$<-0.3$ & 457 & 0.268 & 0.232 & 0.212 & 0.248 & 0.101 & 0.345 & 0.199 & 0.268 \\
{$[-0.3,-0.1)$} & 1657 & 0.568 & 0.415 & 0.391 & 0.425 & 0.141 & 0.420 & 0.259 & 0.636 \\
{$[-0.1,0.1)$} & 2239 & 0.870 & 0.598 & 0.533 & 0.536 & 0.242 & 0.517 & 0.240 & 0.813 \\
{$[0.1,0.3)$} & 1787 & 0.833 & 0.660 & 0.579 & 0.464 & 0.350 & 0.459 & 0.192 & 0.691 \\
$\geq 0.3$ & 1385 & 0.442 & 0.409 & 0.386 & 0.283 & 0.275 & 0.281 & 0.188 & 0.400 \\
\hline
\end{tabular}

(b) 1 month $<\tau<3$ months

\begin{tabular}{cccccccccc} 
Moneyness & $\mathrm{N}$ & SQR & LOU & CTOU & LOUJ & LOUSV & CTOUJ & CTOUSV & CSQR \\
\hline$<-0.3$ & 1970 & 0.304 & 0.224 & 0.222 & 0.231 & 0.159 & 0.347 & 0.244 & 0.380 \\
{$[-0.3,-0.1)$} & 4274 & 0.581 & 0.399 & 0.400 & 0.410 & 0.160 & 0.394 & 0.268 & 0.746 \\
{$[-0.1,0.1)$} & 3966 & 0.814 & 0.515 & 0.507 & 0.513 & 0.199 & 0.496 & 0.178 & 0.792 \\
{$[0.1,0.3)$} & 2272 & 0.968 & 0.663 & 0.640 & 0.510 & 0.373 & 0.505 & 0.202 & 0.765 \\
$\geq 0.3$ & 1505 & 0.651 & 0.512 & 0.498 & 0.333 & 0.346 & 0.321 & 0.218 & 0.538 \\
\hline
\end{tabular}

\begin{tabular}{|c|c|c|c|c|c|c|c|c|c|}
\hline \multicolumn{10}{|c|}{ (c) $\tau>3$ months } \\
\hline Moneyness & $\mathrm{N}$ & SQR & $\mathrm{LOU}$ & CTOU & LOUJ & LOUSV & CTOUJ & CTOUSV & CSQR \\
\hline$<-0.3$ & 1698 & 0.366 & 0.269 & 0.276 & 0.279 & 0.205 & 0.394 & 0.260 & 0.687 \\
\hline$[-0.3,-0.1)$ & 2764 & 0.547 & 0.411 & 0.410 & 0.418 & 0.240 & 0.410 & 0.299 & 0.810 \\
\hline$[-0.1,0.1)$ & 2174 & 0.745 & 0.523 & 0.515 & 0.489 & 0.271 & 0.476 & 0.288 & 0.805 \\
\hline$[0.1,0.3)$ & 990 & 1.068 & 0.746 & 0.730 & 0.556 & 0.404 & 0.542 & 0.344 & 0.920 \\
\hline$\geq 0.3$ & 633 & 0.747 & 0.566 & 0.548 & 0.376 & 0.337 & 0.368 & 0.404 & 0.628 \\
\hline \multicolumn{10}{|c|}{ (d) All maturities } \\
\hline Moneyness & $\mathrm{N}$ & SQR & $\mathrm{LOU}$ & CTOU & LOUJ & LOUSV & CTOUJ & CTOUSV & CSQR \\
\hline$<-0.3$ & 4125 & 0.327 & 0.245 & 0.245 & 0.253 & 0.175 & 0.367 & 0.246 & 0.521 \\
\hline$[-0.3,-0.1)$ & 8695 & 0.568 & 0.406 & 0.402 & 0.415 & 0.186 & 0.404 & 0.277 & 0.748 \\
\hline$[-0.1,0.1)$ & 8379 & 0.812 & 0.540 & 0.516 & 0.513 & 0.231 & 0.497 & 0.228 & 0.801 \\
\hline$[0.1,0.3)$ & 5049 & 0.944 & 0.679 & 0.638 & 0.504 & 0.371 & 0.497 & 0.234 & 0.773 \\
\hline$\geq 0.3$ & 3523 & 0.598 & 0.485 & 0.468 & 0.323 & 0.318 & 0.315 & 0.252 & 0.507 \\
\hline
\end{tabular}

Notes: Moneyness is defined as $\log (K / F(t, T))$, where $K$ and $F(t, T)$ are the strike and futures prices, respectively. "SQR" denotes square root model and "LOU" refers to a log-normal Ornstein-Uhlenbeck process. "LOUJ" introduces jumps in the LOU model, whose size follows an exponential distribution. "CTOU" adds central tendency to the LOU process. "LOUSV" denotes a LOU model with stochastic volatility modelled with a Gamma OU Lévy process. "CTOUJ" adds central tendency and jumps to the LOU model, while "CTOUSV" introduces central tendency and stochastic volatility. "CSQR" is a concatenated SQR model. The column labeled $\mathrm{N}$ gives the number of prices per category. $\tau$ denotes time to maturity. Results are based on full sample estimates. 
Figure 1: Historical evolution of the VIX index

(a) $1990-2010$

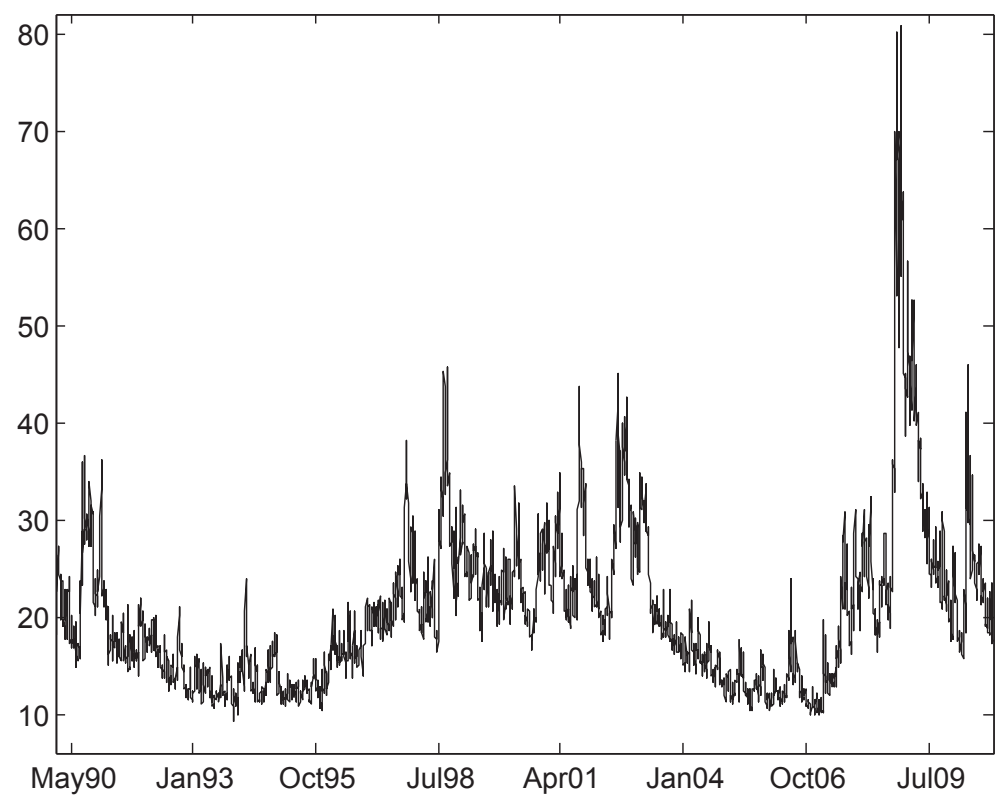

(b) 2006-2010

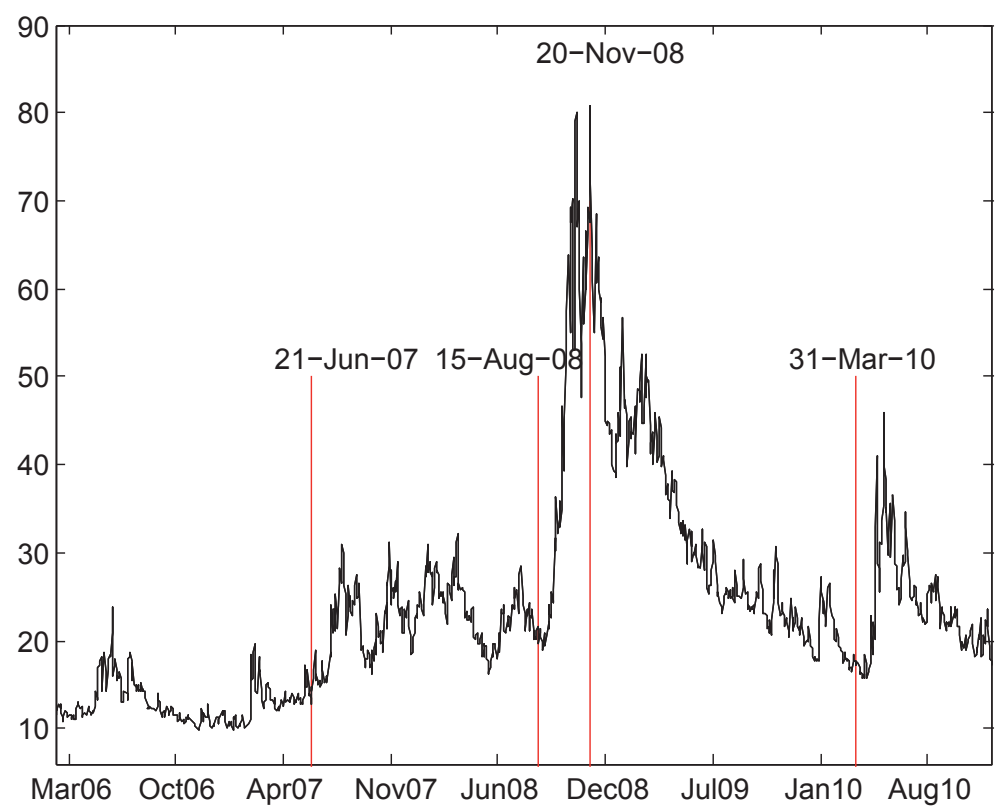


Figure 2: Time series autocorrelations of the log-VIX and estimated ARMA models

(a) Autocorrelations

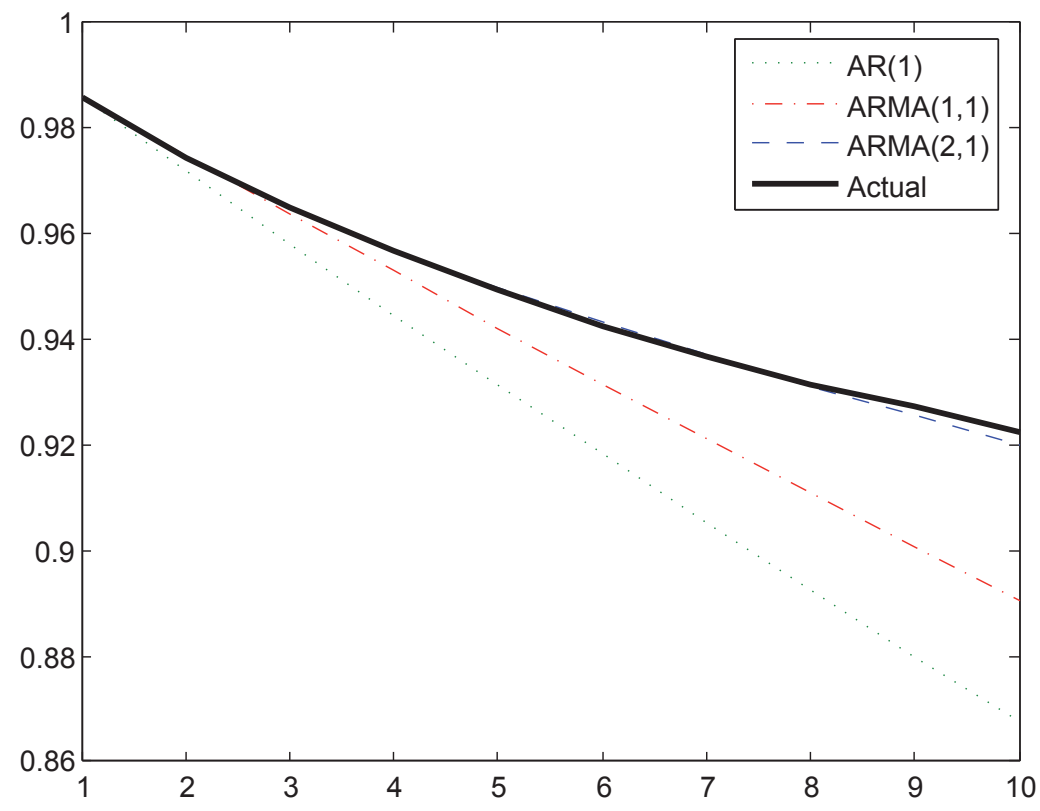

(b) Partial autocorrelations

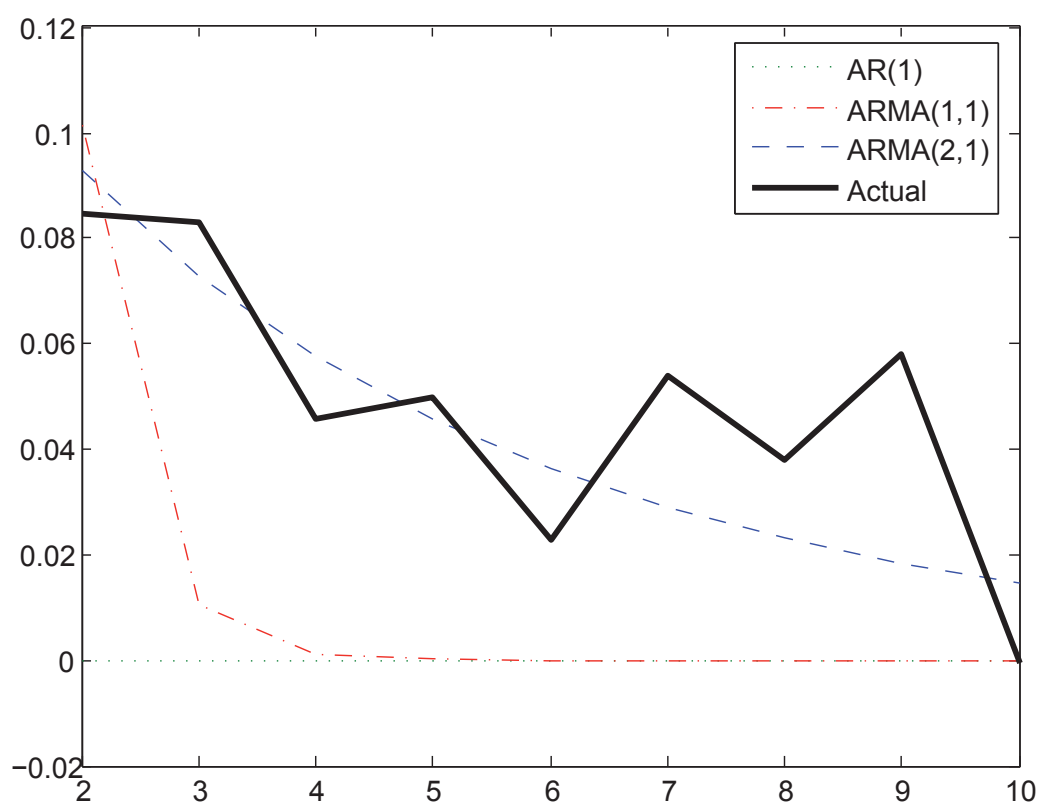

Note: Results are based on the 1990-2010 sample (5280 daily observations). 
Figure 3a: Term structure of VIX futures

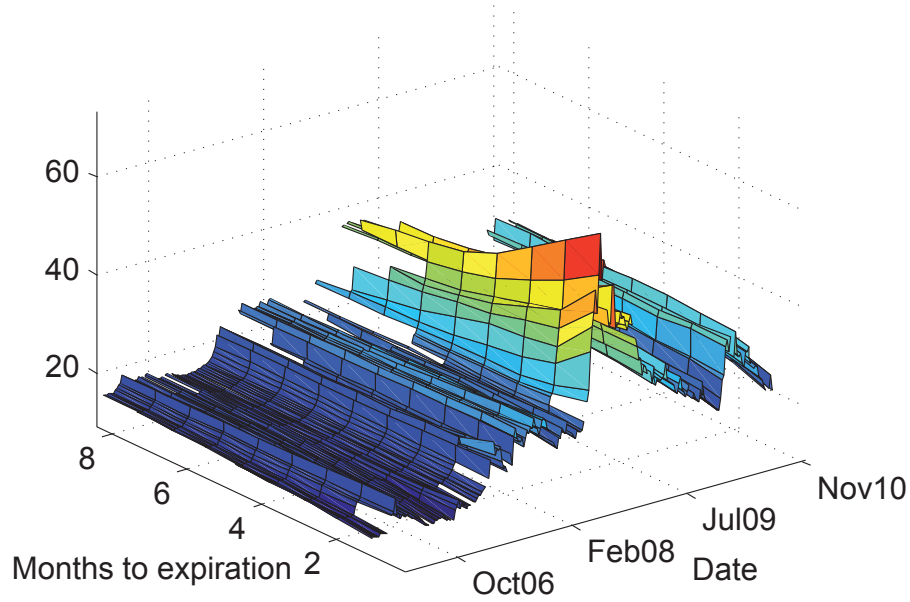

Figure 3b: Implied volatility smirk on low volatility days

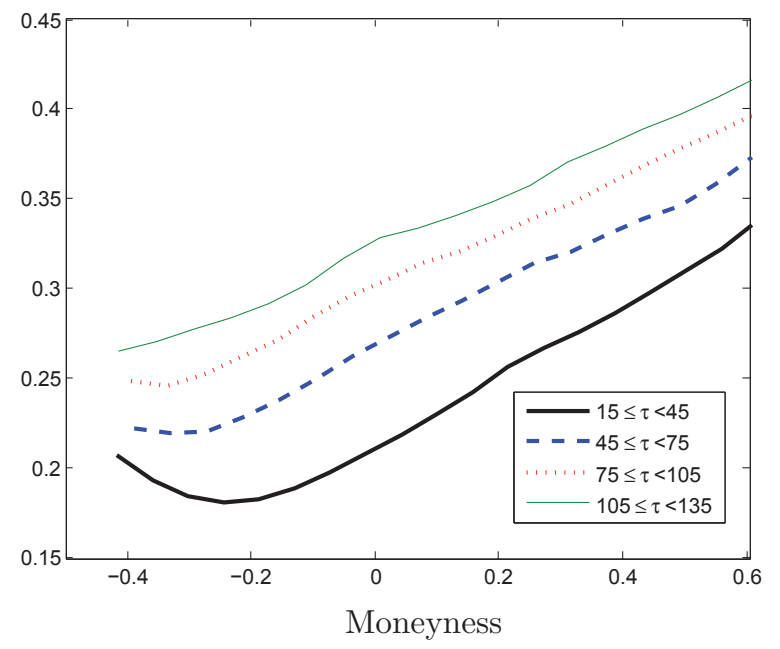

Figure 3c: Implied volatility smirk on high volatility days

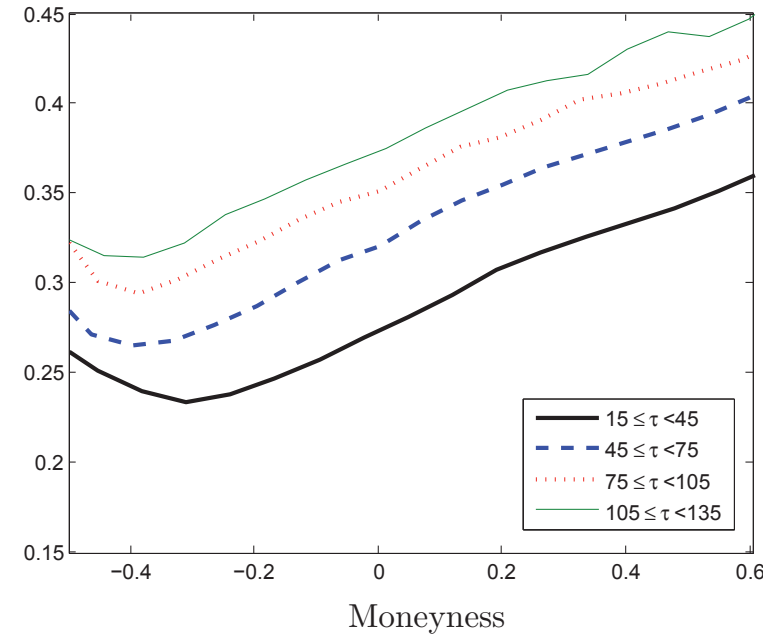

Note: The lines in panels b and c show the average implied volatility for a certain moneyness and time to maturity (denoted by $\tau$ ) within a given interval. Implied volatilities have been obtained by inverting the Black (1976) call price formula. Moneyness is defined as $\log (K / F(t, T)$ ). Low (high) volatility days are those in which one-month at the money implied volatilities are below (above) their average value over the sample. 
Figure 4: Empirical cumulative distribution function of the square pricing errors

(a) Futures

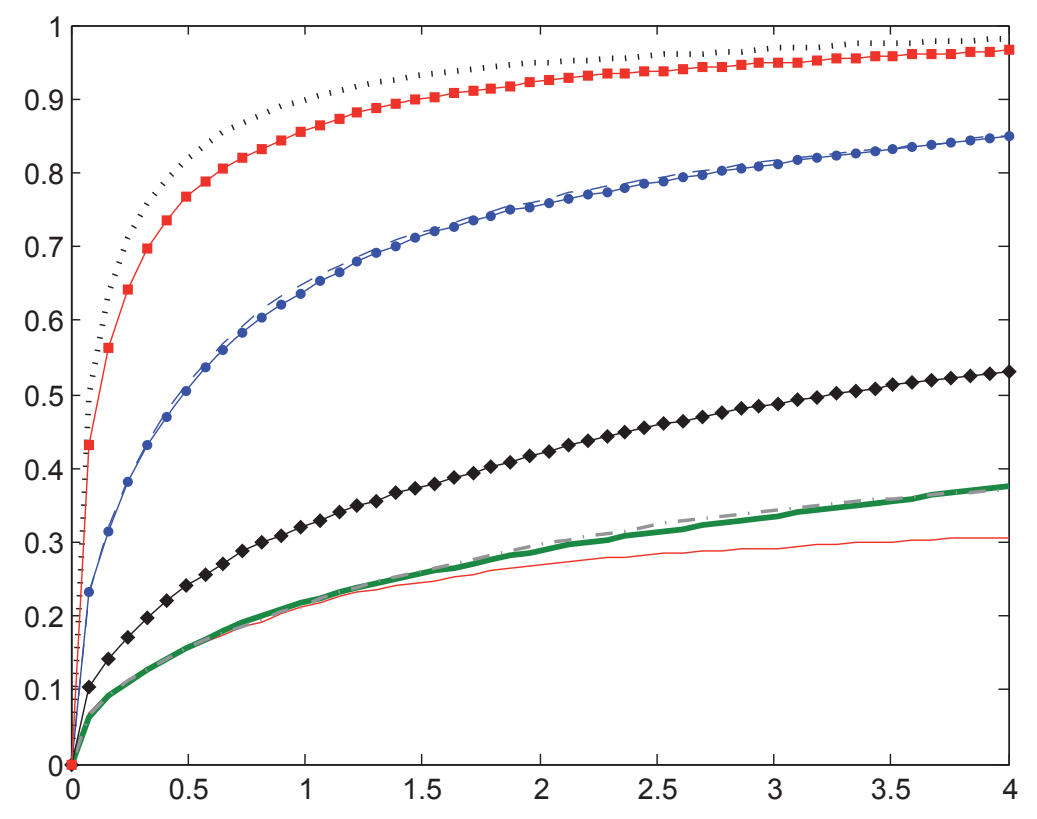

(b) Options

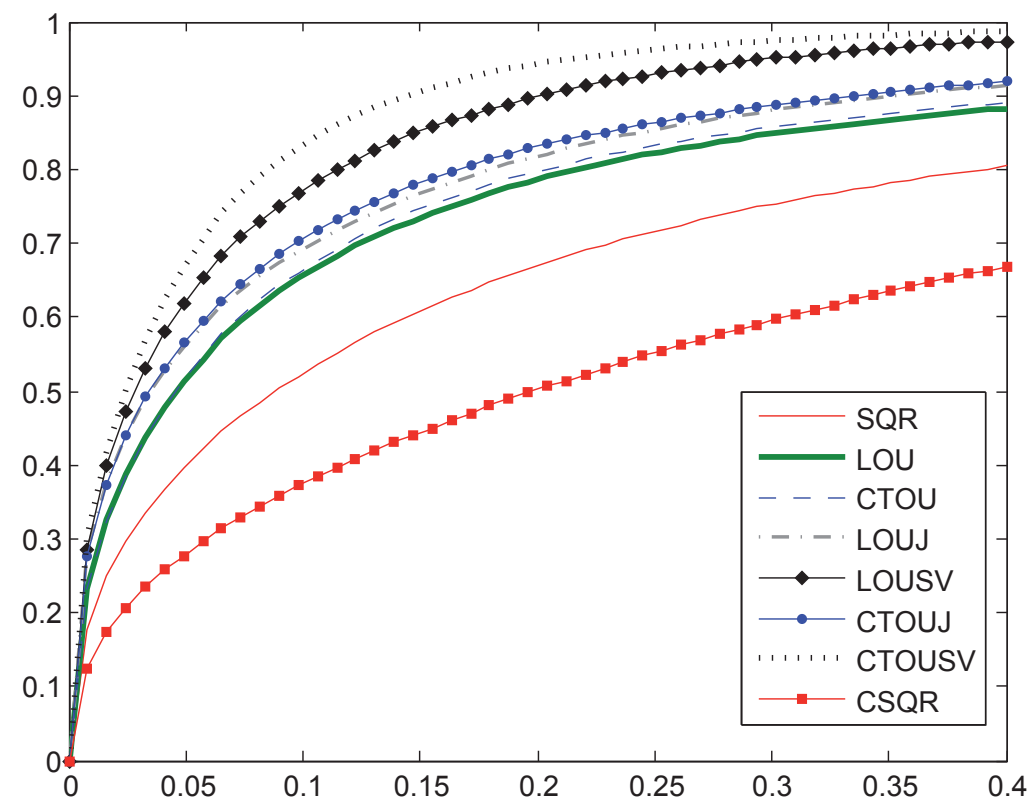

Notes: "SQR" denotes square root model and "LOU" refers to a log-normal Ornstein-Uhlenbeck process. "LOUJ" introduces jumps in the LOU model, whose size follows an exponential distribution. "CTOU" adds central tendency to the LOU process. "LOUSV" denotes a LOU model with stochastic volatility modelled with a Gamma OU Lévy process. "CTOUJ" adds central tendency and jumps to the LOU model, while "CTOUSV" introduces central tendency and stochastic volatility. "CSQR" is the concatenated SQR model. Results are based on full sample estimates. 
Figure 5a: Differences between model-based and actual one-month futures prices

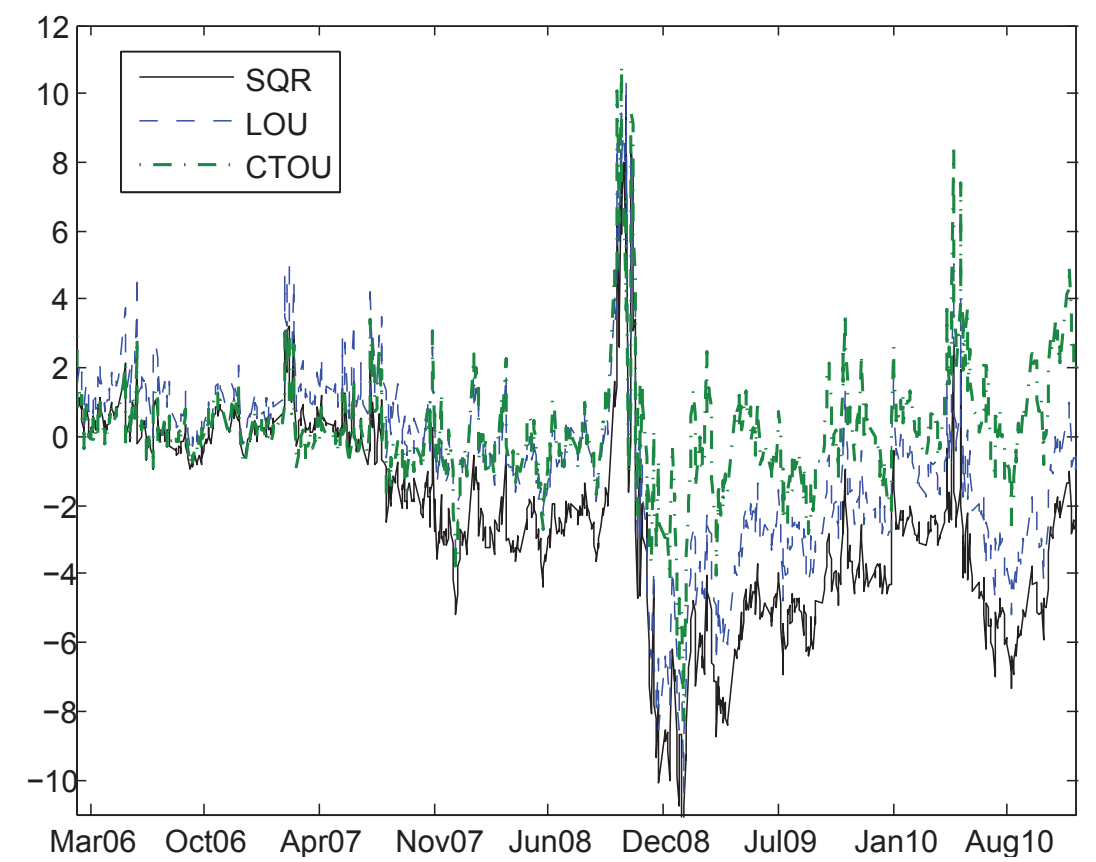

Figure 5b: Differences between model-based and actual one-month futures prices for the two best performing models

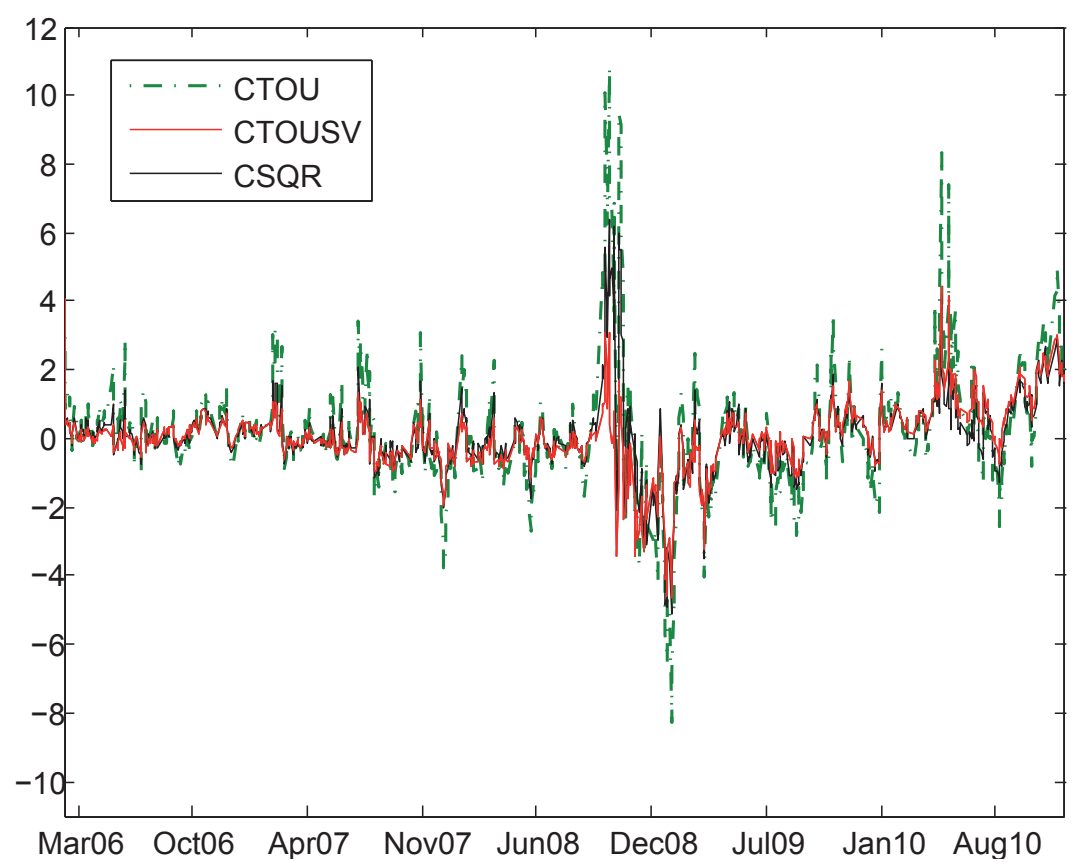

Notes: "SQR" denotes square root model and "LOU" refers to a log-normal Ornstein-Uhlenbeck process. "CTOU" adds central tendency to the LOU process. "CTOUSV" introduces central tendency and stochastic volatility in the LOU model. "CSQR" is the concatenated SQR model. Results are based on full sample estimates. One month actual futures prices have been obtained by interpolation of the prices of the adjacent maturities. 
Figure 6a: Filtered $\theta(t)$ for different $\log -\mathrm{OU}$ models with central tendency

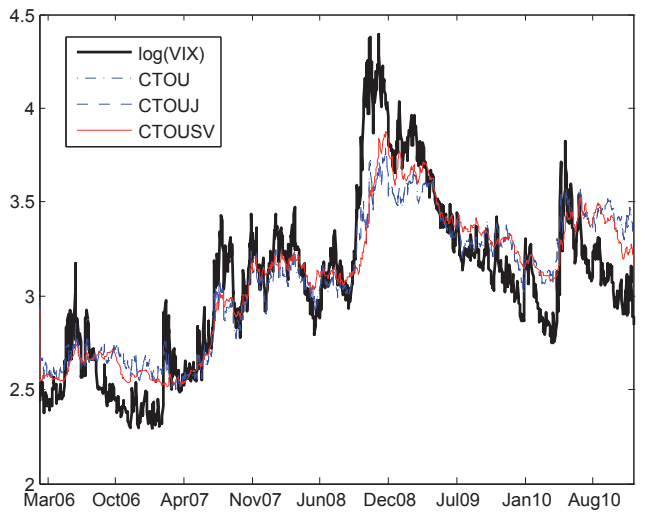

Figure 6c: One month volatilities of the VIX. Risk neutral measure

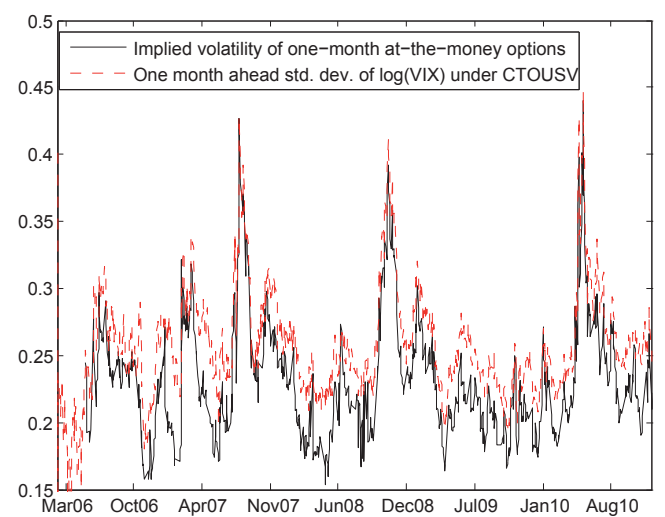

Figure 6b: Filtered $\omega(t)$ for different $\log -\mathrm{OU}$ models with stochastic volatility

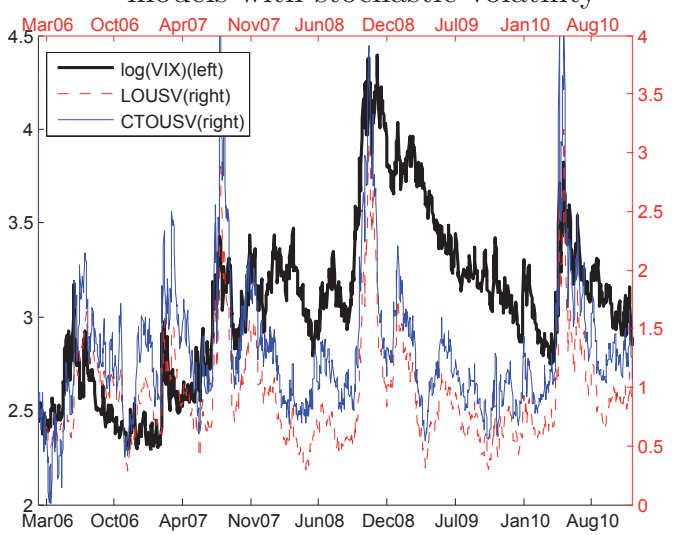

Figure 6d: Logs of one-day ahead standard deviations of the VIX. Real measure

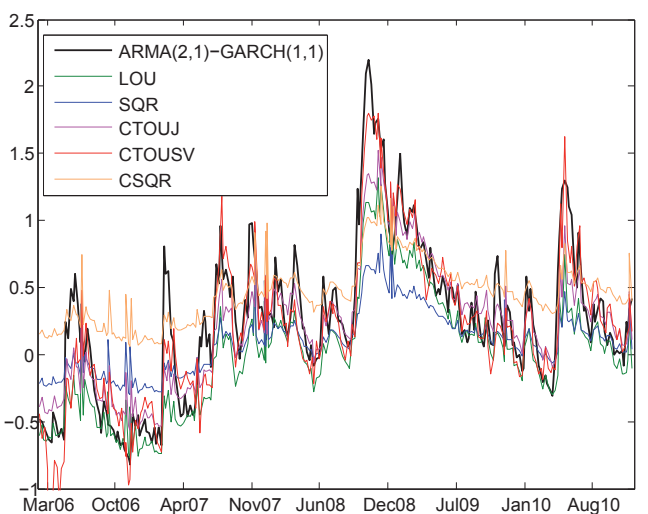

Notes: $\theta(t)$ denotes the time varying central tendency around which the VIX mean-reverts in central tendency models. $\omega(t)$ denotes the instantaneous volatility of stochastic volatility models. Different vertical scales are used for the VIX and the volatilities in panel b. "SQR" denotes square root model and "LOU" refers to a log-normal Ornstein-Uhlenbeck process. "LOUSV" denotes a LOU model with stochastic volatility modelled with a Gamma OU Lévy process. "CTOUJ" adds central tendency and jumps to the LOU model, while "CTOUSV" introduces central tendency and stochastic volatility. "CSQR" is the concatenated SQR model. One-month implied vols in panel c have been obtained by interpolation of the implied vols of options with moneyness $|\log (K / F(t, T))|<.1$, which in turn result from inverting the Black (1976) call price formula. The black line in panel $d$ is the conditional standard deviation of the VIX, obtained from an $\operatorname{ARMA}(2,1)-\operatorname{GARCH}(1,1)$ model estimated for the daily data of the log VIX. Only Thursdays are plotted on panel d to avoid cluttering the picture. Results are based on full sample estimates. 
Figure 7: Fit of the term structure of futures prices

(a) 21-June-2007

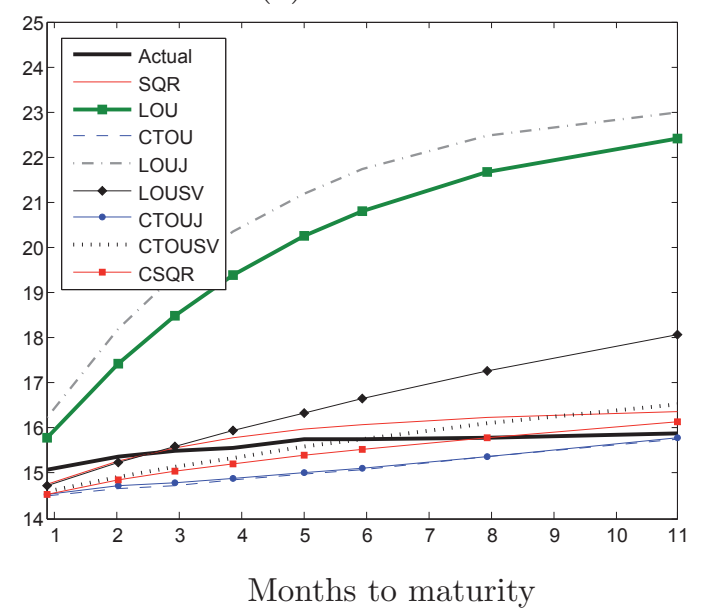

(c) 20-November-2008

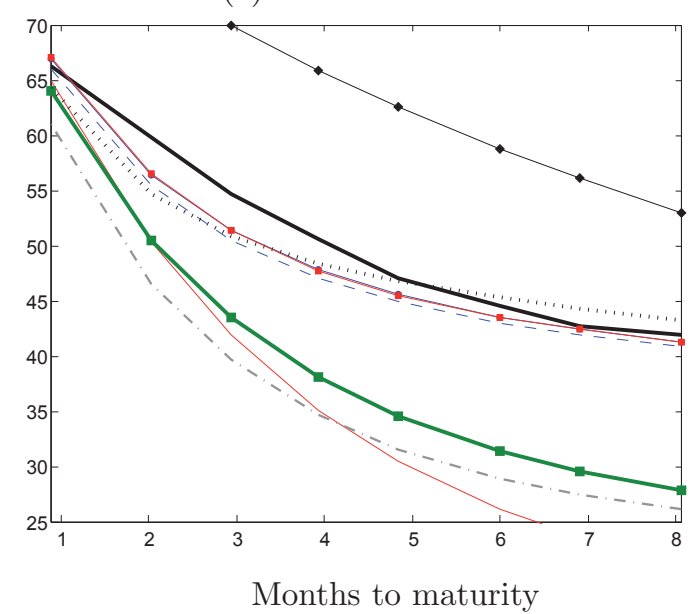

(b) 15-August-2008

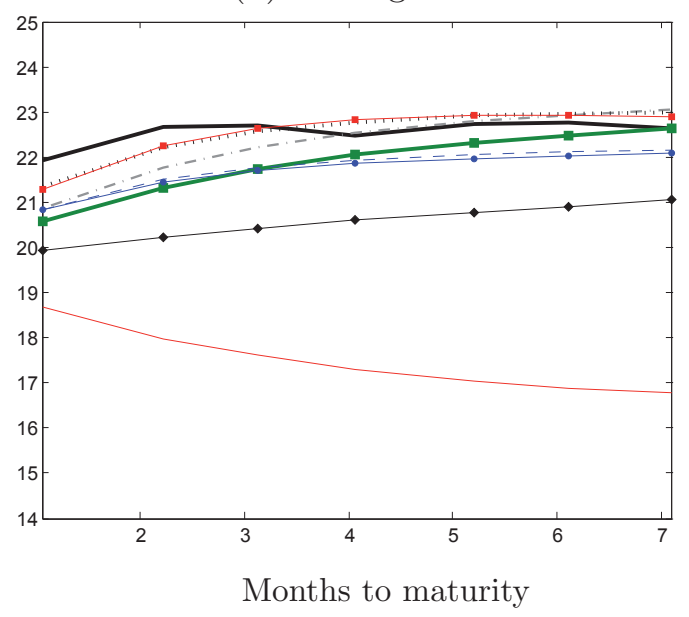

(d) 31-March-2010

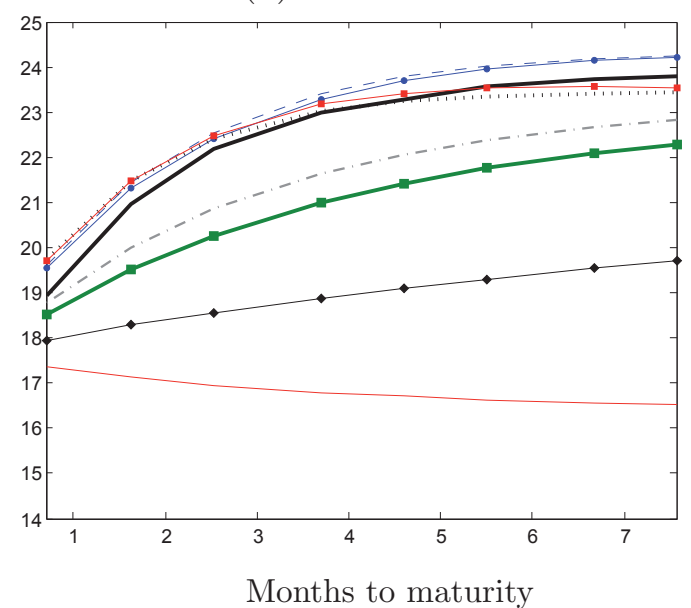

Notes: "SQR" denotes square root model and "LOU" refers to a log-normal Ornstein-Uhlenbeck process. "LOUJ" introduces jumps in the LOU model, whose size follows an exponential distribution. "CTOU" adds central tendency to the LOU process. "LOUSV" denotes a LOU model with stochastic volatility modelled with a Gamma OU Lévy process. "CTOUJ" adds central tendency and jumps to the LOU model, while "CTOUSV" introduces central tendency and stochastic volatility. "CSQR" is the concatenated SQR model. Results are based on full sample estimates. 
Figure 8.1: Fit of the implied volatility smirks. Short maturities

(a) Low volatility. $15 \leq \tau<45$

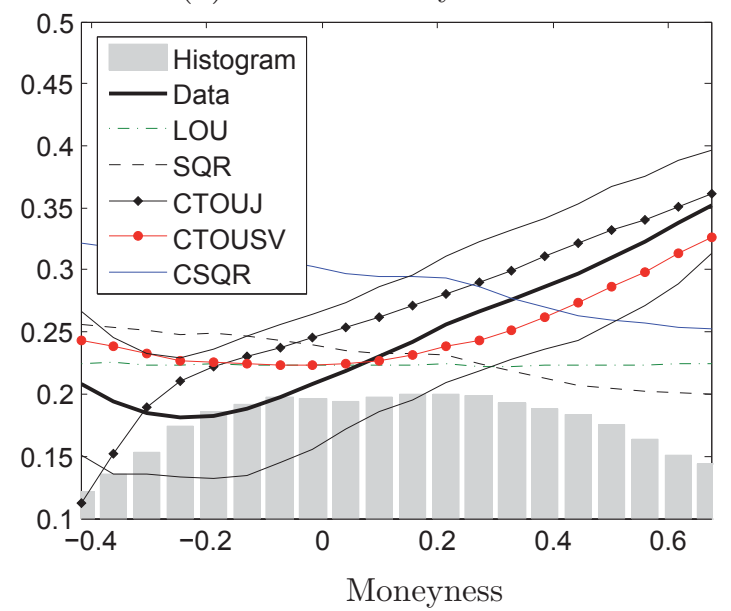

(c) Low volatility. $45 \leq \tau<75$

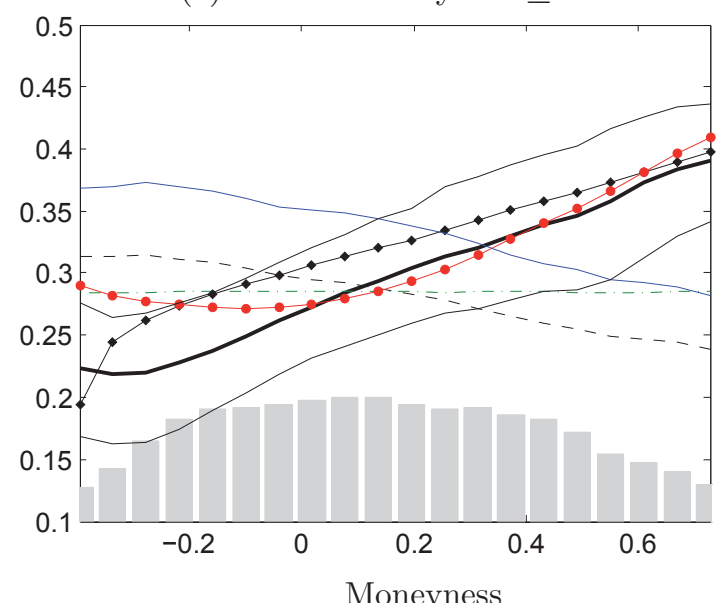

(b) High volatility. $15 \leq \tau<45$

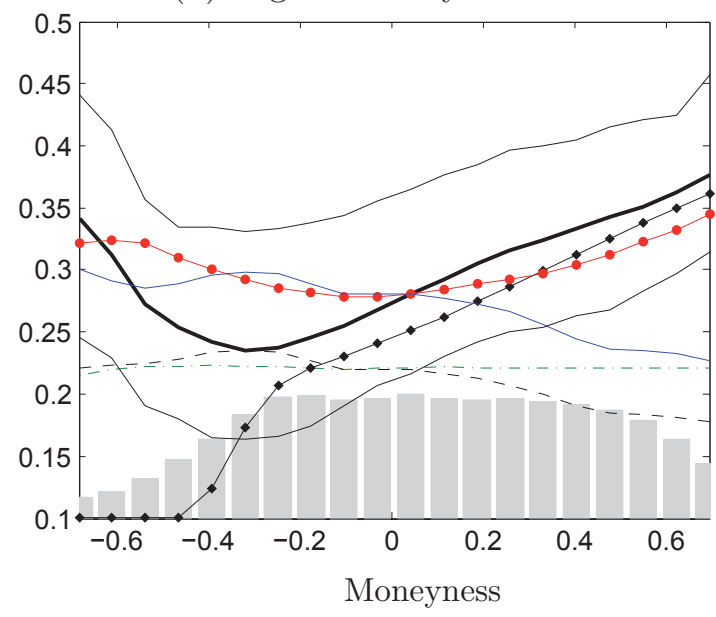

(d) High volatility. $45 \leq \tau<75$

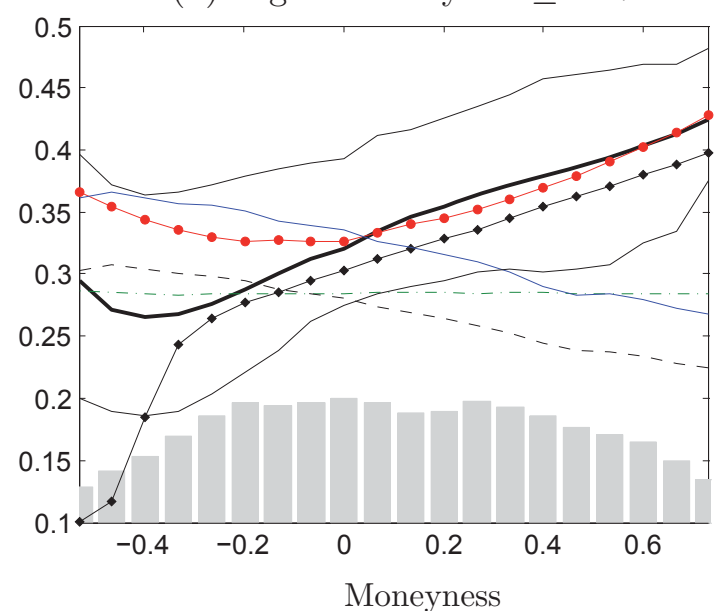

Notes: The lines show the average implied volatility for a certain moneyness and time to maturity (denoted by $\tau$ ) within a given interval. The thick black lines correspond to the average implied volatilities of the actual call prices, while the thin black lines show the $5 \%$ and $95 \%$ percentiles. Grey bars at the bottom of the plots show the histogram of call prices across maturities. The interval of moneyness in each panel has been chosen to cover the central $90 \%$ section of the data. "SQR" denotes square root model and "LOU" refers to a log-normal Ornstein-Uhlenbeck process. "CTOUJ" adds central tendency and jumps to the LOU model, while "CTOUSV" introduces central tendency and stochastic volatility. "CSQR" is the concatenated SQR model. Moneyness is defined as $\log (K / F(t, T)$. Results are based on full sample estimates. 
Figure 8.2: Fit of the implied volatility smirks. Long maturities

(a) Low volatility. $75 \leq \tau<105$

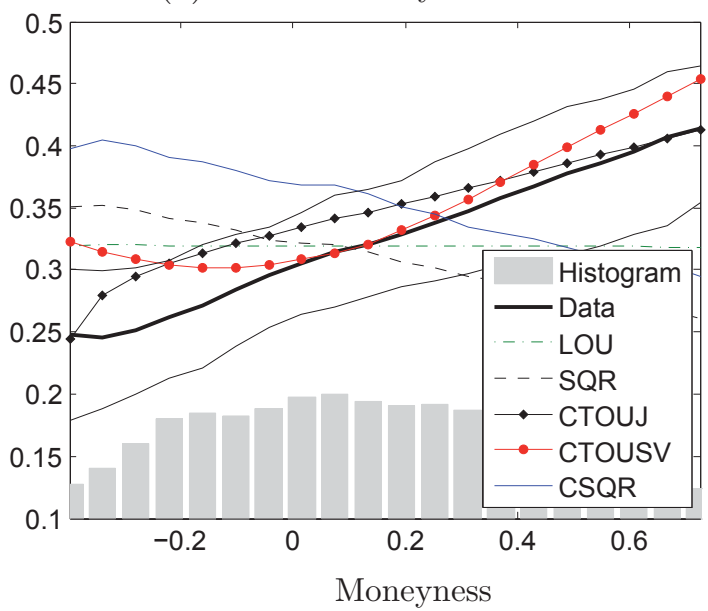

(c) Low volatility. $105 \leq \tau<135$

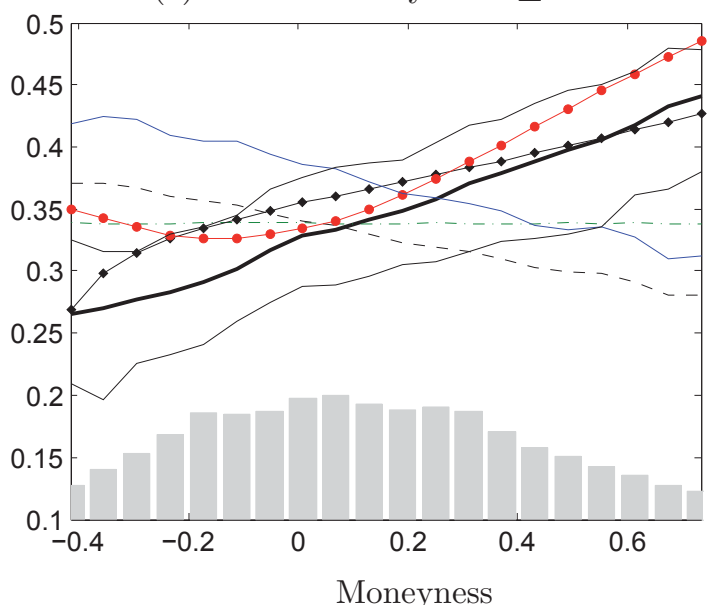

(b) High volatility. $75 \leq \tau<105$

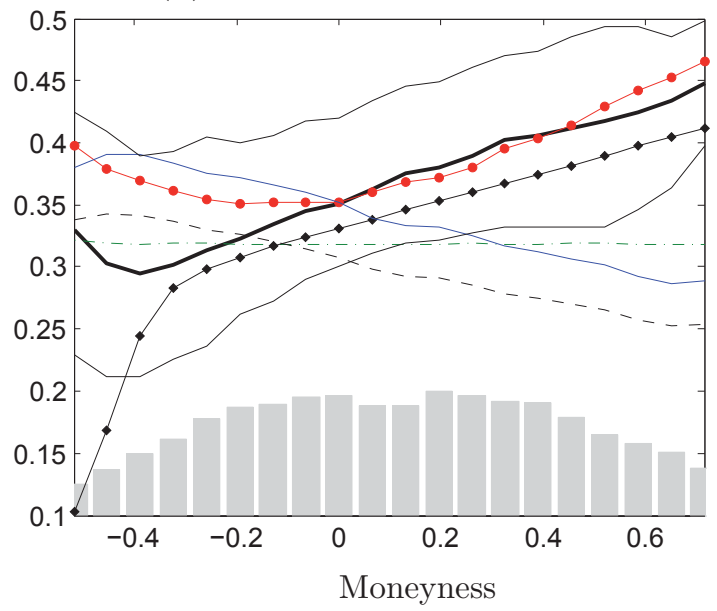

(d) High volatility. $105 \leq \tau<135$

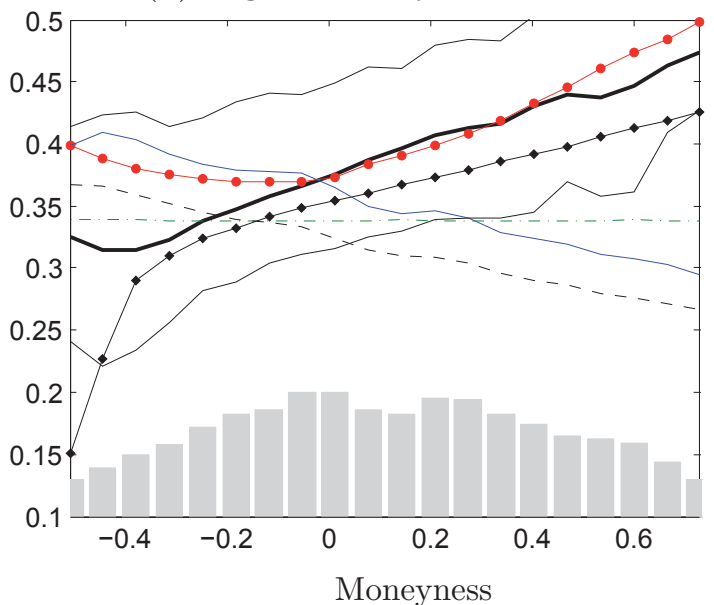

Notes: The lines show the average implied volatility for a certain moneyness and time to maturity (denoted by $\tau$ ) within a given interval. The thick black lines correspond to the average implied volatilities of the actual call prices, while the thin black lines show the $5 \%$ and $95 \%$ percentiles. Grey bars at the bottom of the plots show the histogram of call prices across maturities. The interval of moneyness in each panel has been chosen to cover the central $90 \%$ section of the data. "SQR" denotes square root model and "LOU" refers to a log-normal Ornstein-Uhlenbeck process. "CTOUJ" adds central tendency and jumps to the LOU model, while "CTOUSV" introduces central tendency and stochastic volatility. "CSQR" is the concatenated SQR model. Moneyness is defined as $\log (K / F(t, T)$. Results are based on full sample estimates. 
BANCO DE ESPAÑA PUBLICATIONS

\author{
WORKING PAPERS
}

1101 GIACOMO MASIER and ERNESTO VILLANUEVA: Consumption and initial mortgage conditions: evidence from survey data.

1102 PABLO HERNÁNDEZ DE COS and ENRIQUE MORAL-BENITO: Endogenous fiscal consolidations

1103 CÉSAR CALDERÓN, ENRIQUE MORAL-BENITO and LUIS SERVÉN: Is infrastructure capital productive? A dynamic heterogeneous approach.

1104 MICHAEL DANQUAH, ENRIQUE MORAL-BENITO and BAZOUMANA OUATTARA: TFP growth and its determinants: nonparametrics and model averaging.

1105 JUAN CARLOS BERGANZA and CARMEN BROTO: Flexible inflation targets, forex interventions and exchange rate volatility in emerging countries.

1106 FRANCISCO DE CASTRO, JAVIER J. PÉREZ and MARTA RODRÍGUEZ VIVES: Fiscal data revisions in Europe.

1107 ANGEL GAVILÁN, PABLO HERNÁNDEZ DE COS, JUAN F. JIMENO and JUAN A. ROJAS: Fiscal policy, structural reforms and external imbalances: a quantitative evaluation for Spain.

1108 EVA ORTEGA, MARGARITA RUBIO and CARLOS THOMAS: House purchase versus rental in Spain.

1109 ENRIQUE MORAL-BENITO: Dynamic panels with predetermined regressors: likelihood-based estimation and Bayesian averaging with an application to cross-country growth.

1110 NIKOLAI STÄHLER and CARLOS THOMAS: FiMod - a DSGE model for fiscal policy simulations.

1111 ÁLVARO CARTEA and JOSÉ PENALVA: Where is the value in high frequency trading?

1112 FILIPA SÁ and FRANCESCA VIANI: Shifts in portfolio preferences of international investors: an application to sovereign wealth funds.

1113 REBECA ANGUREN MARTíN: Credit cycles: Evidence based on a non-linear model for developed countries.

1114 LAURA HOSPIDO: Estimating non-linear models with multiple fixed effects: A computational note.

1115 ENRIQUE MORAL-BENITO and CRISTIAN BARTOLUCCI: Income and democracy: Revisiting the evidence.

1116 AGUSTÍN MARAVALL HERRERO and DOMINGO PÉREZ CAÑETE: Applying and interpreting model-based seasonal adjustment. The euro-area industrial production series.

1117 JULIO CÁCERES-DELPIANO: Is there a cost associated with an increase in family size beyond child investment? Evidence from developing countries.

1118 DANIEL PÉREZ, VICENTE SALAS-FUMÁS and JESÚS SAURINA: Do dynamic provisions reduce income smoothing using loan loss provisions?

1119 GALO NUÑO, PEDRO TEDDE and ALESSIO MORO: Money dynamics with multiple banks of issue: evidence from Spain 1856-1874

1120 RAQUEL CARRASCO, JUAN F. JIMENO and A. CAROLINA ORTEGA: Accounting for changes in the Spanish wage distribution: the role of employment composition effects.

1121 FRANCISCO DE CASTRO and LAURA FERNÁNDEZ-CABALLERO: The effects of fiscal shocks on the exchange rate in Spain.

1122 JAMES COSTAIN and ANTON NAKOV: Precautionary price stickiness.

1123 ENRIQUE MORAL-BENITO: Model averaging in economics.

1124 GABRIEL JIMÉNEZ, ATIF MIAN, JOSÉ-LUIS PEYDRÓ AND JESÚS SAURINA: Local versus aggregate lending channels: the effects of securitization on corporate credit supply.

1125 ANTON NAKOV and GALO NUÑO: A general equilibrium model of the oil market.

1126 DANIEL C. HARDY and MARÍA J. NIETO: Cross-border coordination of prudential supervision and deposit guarantees.

1127 LAURA FERNÁNDEZ-CABALLERO, DIEGO J. PEDREGAL and JAVIER J. PÉREZ: Monitoring sub-central government spending in Spain.

1128 CARLOS PÉREZ MONTES: Optimal capital structure and regulatory control.

1129 JAVIER ANDRÉS, JOSÉ E. BOSCÁ and JAVIER FERRI: Household debt and labour market fluctuations.

1130 ANTON NAKOV and CARLOS THOMAS: Optimal monetary policy with state-dependent pricing.

1131 JUAN F. JIMENO and CARLOS THOMAS: Collective bargaining, firm heterogeneity and unemployment.

1132 ANTON NAKOV and GALO NUÑO: Learning from experience in the stock market.

1133 ALESSIO MORO and GALO NUÑO: Does TFP drive housing prices? A growth accounting exercise for four countries.

1201 CARLOS PÉREZ MONTES: Regulatory bias in the price structure of local telephone services. 
1202 MAXIMO CAMACHO, GABRIEL PEREZ-QUIROS and PILAR PONCELA: Extracting non-linear signals from several economic indicators.

1203 MARCOS DAL BIANCO, MAXIMO CAMACHO and GABRIEL PEREZ-QUIROS: Short-run forecasting of the euro-dollar exchange rate with economic fundamentals.

1204 ROCIO ALVAREZ, MAXIMO CAMACHO and GABRIEL PEREZ-QUIROS: Finite sample performance of small versus large scale dynamic factor models.

1205 MAXIMO CAMACHO, GABRIEL PEREZ-QUIROS and PILAR PONCELA: Markov-switching dynamic factor models in real time.

1206 IGNACIO HERNANDO and ERNESTO VILLANUEVA: The recent slowdown of bank lending in Spain: are supply-side factors relevant?

1207 JAMES COSTAIN and BEATRIZ DE BLAS: Smoothing shocks and balancing budgets in a currency union.

1208 AITOR LACUESTA, SERGIO PUENTE and ERNESTO VILLANUEVA: The schooling response to a sustained Increase in low-skill wages: evidence from Spain 1989-2009.

1209 GABOR PULA and DANIEL SANTABÁRBARA: Is China climbing up the quality ladder?

1210 ROBERTO BLANCO and RICARDO GIMENO: Determinants of default ratios in the segment of loans to households in Spain.

1211 ENRIQUE ALBEROLA, AITOR ERCE and JOSÉ MARÍA SERENA: International reserves and gross capital flows. Dynamics during financial stress.

1212 GIANCARLO CORSETTI, LUCA DEDOLA and FRANCESCA VIANI: The international risk-sharing puzzle is at businesscycle and lower frequency.

1213 FRANCISCO ALVAREZ-CUADRADO, JOSE MARIA CASADO, JOSE MARIA LABEAGA and DHANOOS SUTTHIPHISAL: Envy and habits: panel data estimates of interdependent preferences.

1214 JOSE MARIA CASADO: Consumption partial insurance of Spanish households.

1215 J. ANDRÉS, J. E. BOSCÁ and J. FERRI: Household leverage and fiscal multipliers.

1216 JAMES COSTAIN and BEATRIZ DE BLAS: The role of fiscal delegation in a monetary union: a survey of the political economy issues.

1217 ARTURO MACÍAS and MARIANO MATILLA-GARCÍA: Net energy analysis in a Ramsey-Hotelling growth model.

1218 ALFREDO MARTÍN-OLIVER, SONIA RUANO and VICENTE SALAS-FUMÁS: Effects of equity capital on the interest rate and the demand for credit. Empirical evidence from Spanish banks.

1219 PALOMA LÓPEZ-GARCÍA, JOSÉ MANUEL MONTERO and ENRIQUE MORAL-BENITO: Business cycles and investment in intangibles: evidence from Spanish firms.

1220 ENRIQUE ALBEROLA, LUIS MOLINA andPEDRO DEL RÍO: Boom-bust cycles, imbalances and discipline in Europe.

1221 CARLOS GONZÁLEZ-AGUADO and ENRIQUE MORAL-BENITO: Determinants of corporate default: a BMA approach.

1222 GALO NUÑO and CARLOS THOMAS: Bank leverage cycles.

1223 YUNUS AKSOY and HENRIQUE S. BASSO: Liquidity, term spreads and monetary policy.

1224 FRANCISCO DE CASTRO and DANIEL GARROTE: The effects of fiscal shocks on the exchange rate in the EMU and differences with the US.

1225 STÉPHANE BONHOMME and LAURA HOSPIDO: The cycle of earnings inequality: evidence from Spanish social security data.

1226 CARMEN BROTO: The effectiveness of forex interventions in four Latin American countries.

1227 LORENZO RICCI and DAVID VEREDAS: TailCoR.

1228 YVES DOMINICY, SIEGFRIED HÖRMANN, HIROAKI OGATA and DAVID VEREDAS: Marginal quantiles for stationary processes.

1229 MATTEO BARIGOZZI, ROXANA HALBLEIB and DAVID VEREDAS: Which model to match?

1230 MATTEO LUCIANI and DAVID VEREDAS: A model for vast panels of volatilities.

1231 AITOR ERCE: Does the IMF's official support affect sovereign bond maturities?

1232 JAVIER MENCÍA and ENRIQUE SENTANA: Valuation of VIX derivatives.

\section{BANCODEESPAÑA}

Eurosistema
Unidad de Servicios Auxiliares

Alcalá, 48 - 28014 Madrid

Telephone +34913386360

E-mail: publicaciones@bde.es www.bde.es 